\title{
Vertical distribution and diurnal migration of atlantid heteropods
}

\author{
Deborah Wall-Palmer ${ }^{1,7, *}$, Brett Metcalfe ${ }^{2,3}$, Melanie J. Leng ${ }^{4,5}$, Hilary J. Sloane $^{5}$, \\ Gerald Ganssen $^{2}$, P. N. Vinayachandran ${ }^{6}$, Christopher W. Smart ${ }^{1}$ \\ ${ }^{1}$ School of Geography, Earth and Environmental Sciences, University of Plymouth, Plymouth PL4 8AA, UK \\ ${ }^{2}$ Earth and Climate Cluster, Department of Earth Sciences, Faculty of Sciences, Vrije Universiteit Amsterdam, Amsterdam, \\ The Netherlands \\ ${ }^{3}$ Laboratoire des Sciences du Climat et de l'Environnement, LSCE/IPSL, CEA-CNRS-UVSQ, Université Paris-Saclay, \\ 91191 Gif-sur-Yvette, France \\ ${ }^{4}$ School of Biosciences, University of Nottingham, Sutton Bonington Campus, Loughborough LE12 5RD, UK \\ ${ }^{5}$ NERC Isotope Geosciences Facilities, British Geological Survey, Keyworth NG12 5GG, UK \\ ${ }^{6}$ Centre for Atmospheric and Oceanic Sciences, Indian Institute of Science, Bangalore 560 012, India \\ ${ }^{7}$ Present address: Naturalis Biodiversity Center, 2300 RA Leiden, The Netherlands
}

\begin{abstract}
Understanding the vertical distribution and migratory behaviour of shelled holoplanktonic gastropods is essential in determining the environmental conditions to which they are exposed. This is increasingly important in understanding the effects of ocean acidification and climate change. Here we investigated the vertical distribution of atlantid heteropods by collating data from publications and collections and using the oxygen isotope $\left(\delta^{18} \mathrm{O}\right)$ composition of single aragonitic shells. Data from publications and collections show 2 patterns of migration behaviour: small species that reside in shallow water at all times, and larger species that make diurnal migrations from the surface at night to deep waters during the daytime. The $\delta^{18} \mathrm{O}$ data show that all species analysed $(n=16)$ calcify their shells close to the deep chlorophyll maximum. This was within the upper $110 \mathrm{~m}$ of the ocean for 15 species, and down to $146 \mathrm{~m}$ for a single species. These findings confirm that many atlantid species are exposed to large environmental variations over a diurnal cycle and may already be well adapted to face ocean changes. However, all species analysed rely on aragonite supersaturated waters in the upper $<150 \mathrm{~m}$ of the ocean to produce their shells, a region that is projected to undergo the earliest and greatest changes in response to increased anthropogenic $\mathrm{CO}_{2}$.
\end{abstract}

KEY WORDS: Atlantidae - Gastropod • Vertical distribution - Diurnal migration - Oxygen isotopes · Calcification $\cdot$ Ocean acidification

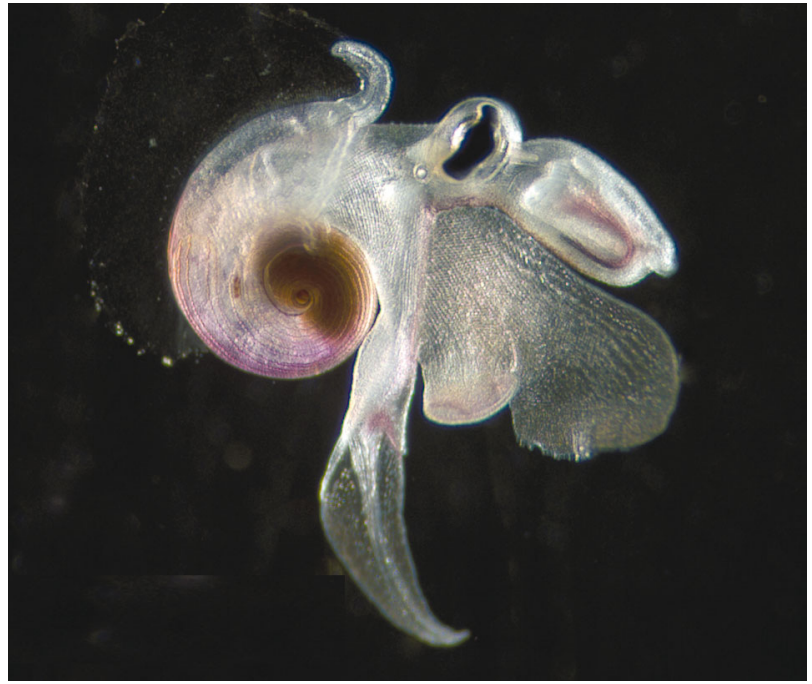

Young adult atlantid heteropod Oxygyrus inflatus collected in the Atlantic Ocean during cruise AMT27. Maximum shell diameter (excluding keel) $1.6 \mathrm{~mm}$.

Photo: @ K. Peijnenburg, E. Goetze, D. Wall-Palmer, L. Mekkes

\section{INTRODUCTION}

Holoplanktonic gastropods spend their entire lives as drifting plankton, unable to swim against currents, but capable of adjusting their vertical position in the water column (Lalli \& Gilmer 1989). This allows them to sink to depths, rise back to the surface or remain at a preferential depth. However, it is still unclear to what 
degree holoplanktonic gastropods take advantage of this vertical freedom, for example, to avoid predation, to reduce competition on finite food resources or to seek preferential environmental conditions.

Two groups of holoplanktonic gastropods are recognized: the pteropods (Thecosomata and Gymnosomata) and the heteropods (Pterotracheoidea). Both groups contain shelled, partially shelled and shellless species, although even shell-less species have a shell in the larval stage, which is subsequently discarded (Lalli \& Gilmer 1989). Holoplanktonic gastropod shells are composed of aragonite, a form of calcium carbonate that is susceptible to dissolution in waters undersaturated with respect to aragonite (Mucci 1983). These sensitive shells have highlighted the euthecosome (fully shelled) pteropods as being amongst the most vulnerable organisms to ocean acidification, with effects already being detected in field populations (Bednaršek \& Ohman 2015). Ocean acidification research has not yet included the heteropods, which also rely on aragonite shells and inhabit the upper ocean, a realm of highly variable environmental parameters. This region will be greatly affected by ocean acidification and climate change, so it is beneficial to understand the vertical distribution of holoplanktonic gastropods. This will help scientists to determine the environmental conditions to which they are frequently exposed and may demonstrate, for example, that holoplanktonic gastropods are already exposed on a daily basis to undersaturated waters, with respect to aragonite, or to temperatures more variable than those predicted to affect oceans over the next 100 yr. Ultimately, holoplanktonic gastropods may already have mechanisms for dealing with a changing ocean (e.g. Maas et al. 2012). Conversely, ocean changes may present even more severe vertical environmental gradients than predicted, which holoplanktonic gastropods may be unable to adapt to, potentially forcing them to modify and constrain their vertical movements.

This study focusses on the shelled heteropods, or atlantids (family Atlantidae), which rely on a shell throughout their life. Atlantids are small $(<14 \mathrm{~mm})$ predatory holoplanktonic gastropods that feed on other metazooplankton, including euthecosome pteropods (Lalli \& Gilmer 1989). Atlantids are able to fully retract into their thin-walled (1.5-8 $\mu \mathrm{m}, \mathrm{D}$. Wall-Palmer pers. obs.) aragonite shells, which are generally a broad, flat disk shape that is necessary for efficient swimming. The vertical distribution of atlantids has been speculated upon by many working in the field. It is generally accepted that atlantids live within the upper $250 \mathrm{~m}$ of the water column and often at much shal- lower depths (Lalli \& Gilmer 1989, Seapy 1990, Michel \& Michel 1991, Paulinose et al. 1992, Jivaluk 1998, Ossenbrügger 2010, Lemus-Santana et al. 2014, Wall-Palmer et al. 2016c). However, many atlantids are thought not to be static in their vertical position in the water column, but to undergo some degree of daily vertical migration. Studies based on sampling with plankton nets at different depths suggest that the depth and timing of this vertical migration are not only species specific, but also are specific to the ontogenetic stage and are probably influenced by seasonal changes (Wall-Palmer et al. 2016c and references therein). To date, the most extensive and thorough study of atlantid vertical distributions and migratory behaviour was conducted by Seapy (1990) offshore of Hawaii (USA). Seapy (1990) demonstrated 2 patterns of distribution: (1) small species that remained in shallow water of $<140 \mathrm{~m}$ depth at all times, being active in the daytime and generally inactive at night, and (2) larger species that are inactive at depth during the daytime, but migrate to the surface at night to feed.

The shell geochemistry of some holoplanktonic gastropods has been used successfully to identify the depth at which shells are grown (e.g. Grossman et al. 1986, Juranek et al. 2003, Keul et al. 2017). The ratio of the oxygen isotopes ${ }^{18} \mathrm{O}$ and ${ }^{16} \mathrm{O}\left(\delta^{18} \mathrm{O}\right)$ incorporated into an aragonite shell is a function of the $\delta^{18} \mathrm{O}$ of the water in which a specimen lives $\left(\delta^{18} \mathrm{O}_{\mathrm{w}}\right)$ and temperature (Grossman \& $\mathrm{Ku}$ 1986). The $\delta^{18} \mathrm{O}_{\mathrm{w}}$ is a conservative water mass tracer related to input (e.g. precipitation and meltwater) and output (e.g. evaporation, sea-ice and brine formation), and therefore is directly relatable to seawater salinity (LeGrande \& Schmidt 2006). Temperature plays a dominant role in the fractionation between ${ }^{18} \mathrm{O}$ and ${ }^{16} \mathrm{O}$ during the formation of aragonite. Therefore, the $\delta^{18} \mathrm{O}$ equilibrium $\left(\delta^{18} \mathrm{O}_{\text {eq }}\right)$ at which aragonite is precipitated in seawater can be calculated from salinity and temperature. Euthecosome pteropods and atlantid heteropods calcify at or close to the aragonite-water isotopic equilibrium; therefore, the $\delta^{18} \mathrm{O}$ of their shells directly records the $\delta^{18} \mathrm{O}_{\text {eq }}$ of the water in which they live (Grossman et al. 1986, Juranek et al. 2003, Keul et al. 2017). Depth of calcification can be inferred by comparing the $\delta^{18} \mathrm{O}$ of the specimen to a $\delta^{18} \mathrm{O}_{\text {eq }}$ depth profile of the ambient water in which the specimen lived (calculated using temperature and salinity, LeGrande \& Schmidt 2006). This technique has been used in a single study on atlantids (Grossman et al. 1986). Grossman et al. (1986) analysed 3 species of atlantid, Atlanta inclinata, A. gaudichaudi and an unidentified species. The isotopic compositions of the 3 species were comparable (apart from a single specimen) and indicated calcification in 
the upper $75 \mathrm{~m}$ of the ocean. The geochemical methods of Grossman et al. (1986) have never been repeated or developed upon to include further atlantid species. Here we investigated the depth distribution of atlantid heteropods using 2 approaches. Firstly, sampling depth and time information gathered from publications and from collections was used to infer the likely depth distribution of each species and patterns of diurnal migration. Secondly, by building on the findings of Grossman et al. (1986), $\delta^{18} \mathrm{O}$ values of single shells were used to determine the depth of calcification for 16 atlantid species at different life stages.

\section{MATERIALS AND METHODS}

\section{Vertical distributions from published data and collections}

Depth and (local) time data were gathered for 4086 specimens identified to species level in collections held at Plymouth Marine Laboratory (Plymouth, UK),
Naturalis Biodiversity Center (Leiden, Netherlands), the Natural History Museum (London), the Natural History Museum of Denmark (Copenhagen) and material collected during the SN105 cruise of the OVR 'Sagar Nidhi' and the SO255 cruise of the RV 'Sonne'. Published depth and time data for 718 atlantid specimens were also used (Tesch 1910, Tokioka 1955, Furnestin 1961, Taki \& Okutani 1962, McGowan \& Fraundorf 1966, Van der Spoel \& Troost 1972, Michel \& Michel 1991, Hernández et al. 1993, Seapy \& Richter 1993, Quesquén Liza 2005, De Vera et al. 2006, De Vera \& Seapy 2006, Ayón et al. 2008, Ossenbrügger 2010, Howard et al. 2011, Wall-Palmer et al. 2016a,b, Burridge et al. 2017). The gathered data were from locations worldwide and from all seasons (Fig. 1). Only specimens that were collected live (using plankton nets) were included, and a maximum sampling depth of $600 \mathrm{~m}$ was applied due to poor temporal sample coverage below this depth. Samples collected over a long period of time (over $2 \mathrm{~h}$ ) were also removed from the dataset. Depth measurements represent the maximum depth of each particular

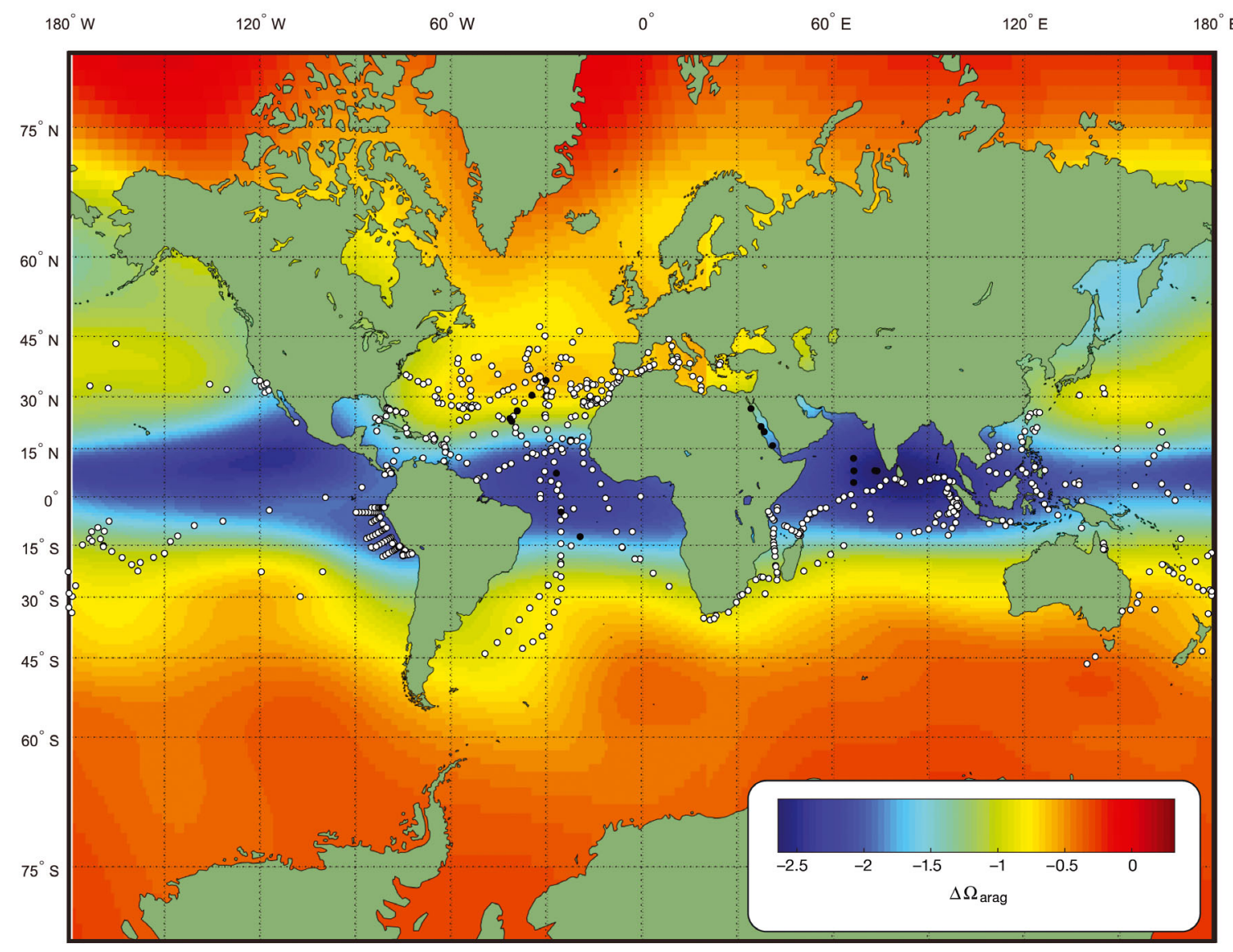

Fig. 1. Collection locations for atlantid specimens used in this study. White dots: specimens examined in collections and data gathered from publications. Black dots: specimens analysed for stable oxygen isotopes to determine the depth of calcification. Base map shows the difference in aragonite saturation state between the surface and $200 \mathrm{~m}$ created using data from Jiang \& Feely (2015) 
sampling. For example, a net may have been towed from $300 \mathrm{~m}$ to the surface, and therefore, specimens may have been caught at any depth between 0 and $300 \mathrm{~m}$. For this reason, the shallowest record for each hourly interval was also determined (Table 1). This indicates the minimum depth at which each species has been found during each time interval.

\section{Depth of calcification stable isotope analysis}

For $\delta^{18} \mathrm{O}$ analysis, a total of 63 specimens from 16 atlantid species were collected using a variety of methods during 4 cruises (Fig. 1): the Atlantic Meridional Transect in 2010 (AMT20) and 2014 (AMT24), the outbound Dutch-Indonesian 'Snellius II' G0 cruise (PG0) and the first cruise of IIOE-2 (SN105). All specimens were fixed and stored in ethanol prior to analysis. Ethanol has been found not to affect either $\delta^{18} \mathrm{O}$ or the carbon isotope composition $\left(\delta^{13} \mathrm{C}\right)$ (Serrano et al. 2008). Specimens were prepared for analysis by rinsing thoroughly in MilliQ water, drying in an oven at $40^{\circ} \mathrm{C}$ and leaving in a desiccator for $24 \mathrm{~h}$. Soft tissues were removed by placing specimens in an Emitech K1050X plasma asher in glass vials for $2.5 \mathrm{~h}$. Plasma ashing does not affect the $\delta^{18} \mathrm{O}$ and $\delta^{13} \mathrm{C}$ of calcium carbonate (Serrano et al. 2008); however, the internal laboratory standard, Keyworth Carrera Marble (KCM), was also plasma ashed to check for any artefacts introduced using this method. The results of plasma ashed and non-plasma ashed KCM were within instrument error. A paired $t$-test found that $\delta^{18} \mathrm{O}$ and $\delta^{13} \mathrm{C}$ values were not significantly different between plasma ashed (pa) and non-plasma-ashed (n-pa) values for $\mathrm{KCM}\left(\delta^{18} \mathrm{O}\right.$ : pa $\mathrm{M}($ mean $)=-1.72$, var $=$ $0.01 ;$ n-pa $\mathrm{M}=-1.73$, var $=0.01 ; t=-0.33, \mathrm{p}=0.75$; $\delta^{13} \mathrm{C}$ : pa $M=2.01$, var $=0.00 ; n-$ pa $M=2.00$, var = 0.00; $t=-0.34, \mathrm{p}=0.74)$, confirming no effect on the isotopic composition from this preparatory technique.

The stable isotope analysis was carried out at the Stable Isotope Facility (part of the NERC Isotope Geoscience Laboratory) at the British Geological Survey. Specimens ranging in weight between 13 and $200 \mu \mathrm{g}$ were analysed individually, although the small size of

Table 1. Maximum depth $(\mathrm{m})$ of shallowest plankton tows collected per hour for each species. Grey shading highlights general periods of darkness. Cells with '- ' denote time periods with no depth data. A. = Atlanta; O. = Oxygyrus; $P$. = Protatlanta

\begin{tabular}{|c|c|c|c|c|c|c|c|c|c|c|c|c|c|c|c|c|c|c|c|c|c|c|c|}
\hline $\begin{array}{l}\text { Time of } \\
\text { day (h) }\end{array}$ & 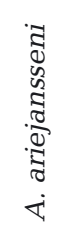 & 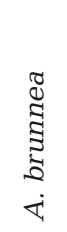 & 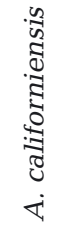 & 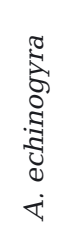 & 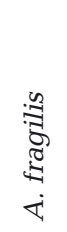 & 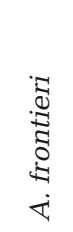 & 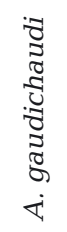 & $\begin{array}{l}\pi \\
0 \\
\delta \\
0 \\
\text { రి } \\
\dot{1}\end{array}$ & 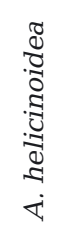 & 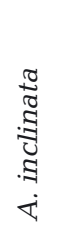 & $\begin{array}{l}\mathbb{\pi} \\
\mathbb{\pi} \\
\mathbb{Z} \\
. \\
\dot{\nabla}\end{array}$ & 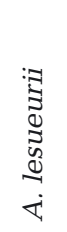 & 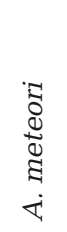 & $\begin{array}{l}\pi \\
5 \\
0 \\
0 \\
: \\
0 \\
0 \\
+1\end{array}$ & 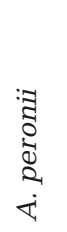 & $\begin{array}{l}\frac{\pi}{\mathcal{H}} \\
\frac{\pi}{\pi} \\
\mathbb{R} \\
\dot{R}\end{array}$ & 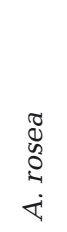 & 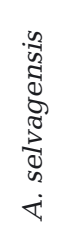 & 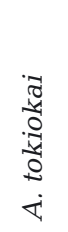 & 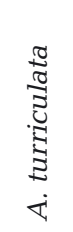 & 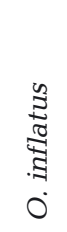 & 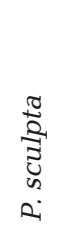 & 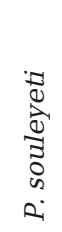 \\
\hline $00: 00-00: 59$ & - & 50 & 15 & 300 & 50 & 100 & 50 & 50 & 50 & 50 & 50 & 50 & - & 300 & 25 & - & - & 200 & 300 & 50 & 10 & 100 & - \\
\hline 01:00-01:59 & 70 & 50 & - & 50 & 400 & 50 & 35 & 50 & 300 & 50 & - & 35 & 600 & 50 & 25 & 50 & 400 & - & 50 & 50 & 0 & - & 50 \\
\hline $02: 00-02: 59$ & - & 50 & - & 50 & 50 & 50 & 50 & 50 & 50 & 50 & 50 & 25 & 200 & 50 & 0 & - & 50 & 100 & 50 & 50 & 0 & 50 & 50 \\
\hline 03:00-03:59 & 216 & 50 & - & 50 & 50 & 50 & 50 & 50 & 50 & 50 & 50 & 0 & 50 & 50 & 25 & 50 & 0 & 100 & 50 & 50 & 50 & 0 & 50 \\
\hline 04:00-04:59 & 200 & 50 & 210 & 50 & 50 & 50 & 50 & 50 & 50 & 50 & 55 & 50 & 50 & 0 & 50 & 50 & 0 & 200 & 50 & 50 & 50 & 50 & 100 \\
\hline 05:00-05:59 & - & 100 & - & 55 & 100 & 55 & 50 & 55 & 55 & 18 & 55 & 18 & - & 55 & 25 & 55 & - & 300 & 18 & 55 & 25 & - & 100 \\
\hline $06: 00-06: 59$ & - & - & - & 100 & 100 & 50 & 10 & - & - & 100 & 10 & 50 & - & 100 & 10 & 50 & 100 & 100 & 100 & 10 & 100 & - & 100 \\
\hline $07: 00-07: 59$ & - & 400 & - & - & - & - & 50 & 10 & 10 & 10 & 400 & 10 & - & - & 10 & - & - & - & 600 & - & 100 & - & 400 \\
\hline 08:00-08:59 & - & - & - & - & - & - & 50 & - & - & 100 & - & 50 & - & - & 32 & - & - & - & - & - & 100 & - & 100 \\
\hline 09:00-09:59 & - & - & - & 396 & 396 & - & 50 & 459 & 396 & - & - & 10 & - & 459 & 32 & - & 396 & - & 396 & 10 & 25 & - & 459 \\
\hline $10: 00-10: 59$ & - & 100 & - & 100 & 200 & 100 & 50 & - & 100 & 150 & 100 & 50 & - & 100 & 32 & 100 & 100 & 100 & 100 & 100 & 50 & - & 100 \\
\hline $11: 00-11: 59$ & - & - & - & 376 & 376 & - & 50 & - & - & - & - & 50 & - & - & 32 & - & 376 & 200 & 376 & 376 & 105 & - & 376 \\
\hline $12: 00-12: 59$ & - & - & - & - & - & - & 50 & - & - & 150 & 140 & 50 & - & - & 31 & - & - & 200 & - & - & 140 & - & - \\
\hline $13: 00-13: 59$ & - & 200 & - & - & - & - & 50 & - & - & 300 & - & 50 & - & - & 32 & - & 100 & 200 & - & - & 110 & - & - \\
\hline $14: 00-14: 59$ & - & 400 & 150 & - & - & - & 50 & 411 & 411 & 366 & 411 & 50 & - & - & 32 & 411 & - & - & 411 & 411 & 2 & - & 200 \\
\hline $15: 00-15: 59$ & - & 140 & - & - & - & - & 140 & - & - & 400 & 140 & 140 & - & 566 & 32 & 566 & - & 100 & - & 140 & 140 & - & - \\
\hline $16: 00-16: 59$ & - & 140 & - & - & 200 & 370 & 50 & 400 & 200 & 600 & 140 & 50 & - & 370 & 140 & - & 370 & 200 & - & 140 & 100 & - & 100 \\
\hline $17: 00-17: 59$ & - & 140 & - & - & - & - & 50 & - & - & 310 & 140 & 10 & - & - & 140 & - & - & - & - & - & 140 & - & - \\
\hline $18: 00-18: 59$ & - & 100 & 10 & 100 & 50 & 50 & 10 & 50 & 50 & 10 & 10 & 10 & 50 & 50 & 10 & 100 & 50 & 11 & 50 & 0 & 0 & 50 & 10 \\
\hline $19: 00-19: 59$ & 20 & 50 & - & 50 & 23 & 50 & 0 & 50 & 50 & 50 & 50 & 23 & 100 & 50 & 23 & 50 & 50 & 80 & 0 & 0 & 10 & 50 & 50 \\
\hline $20: 00-20: 59$ & - & 10 & - & 50 & 15 & 50 & 50 & 50 & 50 & 0 & 10 & 50 & 60 & 0 & 0 & 50 & 50 & 100 & 25 & 50 & 0 & 50 & 10 \\
\hline $21: 00-21: 59$ & - & 50 & - & 50 & 50 & 50 & 50 & 40 & 50 & 19 & 50 & 0 & 50 & 50 & 0 & 50 & 19 & 200 & 50 & 50 & 0 & 19 & 50 \\
\hline $22: 00-22: 59$ & - & 50 & 50 & 50 & 50 & 50 & 50 & 50 & 50 & 50 & 140 & 25 & 50 & 50 & 25 & 100 & 50 & - & 100 & 50 & 50 & - & 50 \\
\hline $23: 00-23: 59$ & - & 50 & 210 & 0 & 100 & 50 & 50 & 50 & 50 & 50 & 0 & 0 & 50 & 50 & 0 & 50 & 50 & - & 100 & 0 & 50 & - & 100 \\
\hline
\end{tabular}


some juvenile specimens meant that up to 3 specimens of a single species were analysed together (Table 2). Analysis was carried out using an IsoPrime dual inlet mass spectrometer with a Multiprep device. Speci- mens were loaded into glass vials and sealed with septa. The automated system first evacuated the ambient air within the vials and subsequently delivered anhydrous phosphoric acid to the carbonate at $90^{\circ} \mathrm{C}$.

Table 2. Stable oxygen isotope analysis of atlantid specimens and the depth of calcification determined by comparison of specimen values to the aragonite equilibrium of the water column. Cells with '- ' denote stations with only a single specimen where mean and SD are not applicable. For a single specimen, the calculated depth of calcification was above sea level and therefore 'not possible'

\begin{tabular}{|c|c|c|c|c|c|c|c|c|c|}
\hline \multirow{2}{*}{$\begin{array}{l}\text { Specimen code } \\
\text { (Species_Cruise_- } \\
\text { Station_Specimen) }\end{array}$} & \multirow[t]{2}{*}{ Species } & \multirow{2}{*}{$\begin{array}{l}\text { Speci- } \\
\text { mens } \\
\text { (n) }\end{array}$} & \multirow{2}{*}{$\begin{array}{l}\text { Description } \\
\text { of } \\
\text { specimens }\end{array}$} & \multirow[t]{2}{*}{ Latitude } & \multirow{2}{*}{ Longitude } & \multicolumn{3}{|c|}{ Specimen $\delta^{18} \mathrm{O}(\%)$} & \multirow{2}{*}{$\begin{array}{c}\text { Depth of } \\
\text { calcification } \\
\text { (m) }\end{array}$} \\
\hline & & & & & & $\begin{array}{l}\text { Calculated as } \\
\text { aragonite }\end{array}$ & $\begin{array}{c}\text { Mean } \\
\text { station }^{-1}\end{array}$ & $\mathrm{SD}$ & \\
\hline Abru_AMT20_07_01 & Atlanta brunnea & 1 & Adult & $34.23^{\circ} \mathrm{N}$ & $29.73^{\circ} \mathrm{W}$ & -0.96 & -0.84 & 0.17 & 46.92 \\
\hline Abru_AMT20_07_02 & & 1 & Adult & & & -0.72 & & & 61.91 \\
\hline Afro_SN105_01_01 & A. frontieri & 1 & Adult & $11.89^{\circ} \mathrm{N}$ & $66.97^{\circ} \mathrm{E}$ & -1.85 & -1.84 & 0.07 & 96.57 \\
\hline Afro_SN105_01_02 & & 1 & Adult & & & -1.91 & & & 91.94 \\
\hline Afro_SN105_01_03 & & 1 & Adult & & & -1.77 & & & 99.31 \\
\hline Agib_SN105_04_01A & A. gibbosa & 0.5 & Juvenile & $8.02^{\circ} \mathrm{N}$ & $67.08^{\circ} \mathrm{E}$ & -1.73 & -1.69 & 0.06 & 88.40 \\
\hline Agib_SN105_04_01B & & 0.5 & Adult whorl only & & & -1.71 & & & 88.91 \\
\hline Agib_SN105_04_02 & & 1 & Adult & & & -1.72 & & & 88.66 \\
\hline Agib_SN105_04_03 & & 1 & Adult & & & -1.60 & & & 93.93 \\
\hline Ahel_PG0_78_01 & A. helicinoidea & 1 & Adult & $26.62^{\circ} \mathrm{N}$ & $34.72^{\circ} \mathrm{E}$ & -0.91 & - & - & 38.95 \\
\hline Ahel_PG0_86_01 & & 1 & Adult & $21.45^{\circ} \mathrm{N}$ & $37.87^{\circ} \mathrm{E}$ & -0.94 & - & - & 63.11 \\
\hline Ahel_PG0_88_01 & & 1 & Adult & $19.93^{\circ} \mathrm{N}$ & $38.87^{\circ} \mathrm{E}$ & -1.10 & - & - & 78.89 \\
\hline Ainc_AMT20_05/11_01 & A. inclinata & 1 & Juvenile & $4.89^{\circ} \mathrm{S}$ & $25.03^{\circ} \mathrm{W}$ & -1.47 & -1.44 & 0.30 & 101.24 \\
\hline Ainc_AMT20_05/11_02 & & 1 & Juvenile & & & -1.64 & & & 97.13 \\
\hline Ainc_AMT20_05/11_03 & & 1 & Juvenile & & & -1.65 & & & 96.69 \\
\hline Ainc_AMT20_05/11_04 & & 1 & Juvenile & & & -1.01 & & & 109.59 \\
\hline Ainf_PG0_141_01 & A. inflata & 1 & Adult & $8.00^{\circ} \mathrm{N}$ & $74.34^{\circ} \mathrm{E}$ & -2.14 & -2.34 & 0.28 & 66.23 \\
\hline Ainf_PG0_141_02 & & 1 & Adult & & & -2.54 & & & 58.45 \\
\hline Ainf_PG0_140_01 & & 1 & Adult & $8.09^{\circ} \mathrm{N}$ & $73.69^{\circ} \mathrm{E}$ & -2.12 & - & - & 66.54 \\
\hline Ales_PG0_94_01 & A. lesueurii & 1 & Adult & $15.78^{\circ} \mathrm{N}$ & $41.51^{\circ} \mathrm{E}$ & -1.84 & -1.79 & 0.29 & 78.16 \\
\hline Ales_PG0_94_02 & & 1 & Adult & & & -2.06 & & & 66.30 \\
\hline Ales_PG0_94_03 & & 1 & Adult & & & -1.48 & & & 106.68 \\
\hline Aoli_SN105_08_01 & A. oligogyra & 1 & Adult & $4.38^{\circ} \mathrm{N}$ & $67.00^{\circ} \mathrm{E}$ & -2.30 & -2.41 & 0.10 & 59.84 \\
\hline Aoli_SN105_08_02 & & 1 & Adult & & & -2.49 & & & 57.70 \\
\hline Aoli_SN105_08_03 & & 1 & Adult & & & -2.44 & & & 58.15 \\
\hline Apla_PG0_94_01 & A. plana & 1 & Adult & $15.78^{\circ} \mathrm{N}$ & $41.51^{\circ} \mathrm{E}$ & -1.79 & -1.81 & 0.02 & 82.54 \\
\hline Apla_PG0_94_02 & & 1 & Adult & & & -1.83 & & & 78.93 \\
\hline Aros_AMT20_23/10_01 & A. rosea & 1 & Adult & $30.29^{\circ} \mathrm{N}$ & $34.17^{\circ} \mathrm{W}$ & -0.42 & -0.49 & 0.10 & 95.50 \\
\hline Aros_AMT20_23/10_02 & & 1 & Adult & & & -0.46 & & & 92.77 \\
\hline Aros_AMT20_23/10_03 & & 1 & Adult & & & -0.60 & & & 80.93 \\
\hline Asel_AMT20_11_01 & A. selvagensis & 1 & Adult & $25.99^{\circ} \mathrm{N}$ & $38.79^{\circ} \mathrm{W}$ & -0.94 & - & - & 74.35 \\
\hline Asel_AMT20_26/10_01 & & 1 & Adult & $22.96^{\circ} \mathrm{N}$ & $40.53^{\circ} \mathrm{W}$ & -1.28 & - & - & Not possible \\
\hline Asel_AMT20_23/10_01 & & 1 & Adult & $30.29^{\circ} \mathrm{N}$ & $34.17^{\circ} \mathrm{W}$ & -0.99 & - & - & 58.55 \\
\hline Atok_AMT20_09_01 & A. tokiokai & 1 & Adult & $30.29^{\circ} \mathrm{N}$ & $34.19^{\circ} \mathrm{W}$ & -0.58 & - & - & 82.51 \\
\hline Atok_AMT20_26/10_01 & & 1 & Juvenile & $22.96^{\circ} \mathrm{N}$ & $40.53^{\circ} \mathrm{W}$ & -1.09 & -1.16 & 0.06 & 81.30 \\
\hline Atok_AMT20_26/10_02 & & 1 & Juvenile & & & -1.22 & & & 72.73 \\
\hline Atok_AMT20_26/10_03 & & 1 & Juvenile & & & -1.17 & & & 77.12 \\
\hline Atur_SN105_01_01 & A. turriculata & 1 & Adult & $11.89^{\circ} \mathrm{N}$ & $66.97^{\circ} \mathrm{E}$ & -1.69 & -1.72 & 0.08 & 100.30 \\
\hline Atur_SN105_01_02 & & 1 & Adult & & & -1.74 & & & 99.69 \\
\hline Atur_SN105_01_03 & & 1 & Adult & & & -1.63 & & & 100.99 \\
\hline Atur_SN105_01_04 & & 3 & Juvenile & & & -1.83 & & & 97.46 \\
\hline Oinf_SN105_08_01 & Oxygyrus inflatus & 2 & Juvenile & $4.38^{\circ} \mathrm{N}$ & $67.00^{\circ} \mathrm{E}$ & -1.90 & -1.83 & 0.07 & 68.05 \\
\hline Oinf_SN105_08_02 & & 1 & Juvenile & & & -1.91 & & & 67.86 \\
\hline Oinf_SN105_08_03 & & 2 & Juvenile & & & -1.77 & & & 72.73 \\
\hline Oinf_SN105_08_04 & & 2 & Juvenile & & & -1.83 & & & 69.58 \\
\hline Oinf_SN105_08_05 & & 2 & Juvenile & & & -1.77 & & & 72.73 \\
\hline Pscu_AMT24_13_01 & Protatlanta sculpta & 4 & Juvenile & $7.29^{\circ} \mathrm{N}$ & $26.49^{\circ} \mathrm{W}$ & -0.32 & -0.38 & 0.08 & 100.56 \\
\hline Pscu_AMT24_13_02 & & 5 & Juvenile & & & -0.44 & & & 93.23 \\
\hline Psou_AMT20_23_01 & P. souleyeti & 1 & Adult & $12.54^{\circ} \mathrm{S}$ & $19.03^{\circ} \mathrm{W}$ & -0.48 & - & - & 145.56 \\
\hline Psou_AMT20_11_01 & & 1 & Adult & $25.99^{\circ} \mathrm{N}$ & $38.79^{\circ} \mathrm{W}$ & -0.72 & - & - & 89.83 \\
\hline Psou_AMT20_12_01 & & 1 & Adult & $23.77^{\circ} \mathrm{N}$ & $41.11^{\circ} \mathrm{W}$ & -0.51 & - & - & 133.06 \\
\hline
\end{tabular}


The evolved $\mathrm{CO}_{2}$ was collected for 15 min, cryogenically trapped, cleaned of impurities and water vapour and passed to the mass spectrometer. Isotope values $\left(\delta^{13} \mathrm{C}\right.$ [not discussed herein], $\left.\delta^{18} \mathrm{O}\right)$ are reported in per mille (\%o) deviations of the isotopic ratios $\left(R={ }^{18} \mathrm{O} /{ }^{16} \mathrm{O}\right)$ against a standard gas calculated to the Vienna PeeDee Belemnite (V-PDB) scale using a withinrun in-house laboratory standard calibrated against the National Bureau of Standards No. 19 (NBS-19). The aragonite-acid fractionation factor (1000 ln

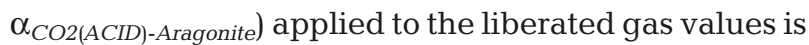
1.00855. Due to the long run time of $21 \mathrm{~h}$, a drift correction was applied across the run, calculated using the KCM standards that bracket the samples. The correction of Craig (1957) was applied to account for ${ }^{17} \mathrm{O}$. The average analytical reproducibility of the standard calcite (KCM) within run was $<0.1 \%$ for $\delta^{18} \mathrm{O}$.

\section{Conversion of water column profiles into $\delta^{18} \mathrm{O}_{\mathrm{eq}}$}

Calcification depth was determined by comparing the measured isotope values to a likely equilibrium oxygen isotope value $\left(\delta^{18} \mathrm{O}_{\text {eq }}\right)$ calculated using salinity and temperature (Grossman et al. 1986, Juranek et al. 2003). $\delta^{18} \mathrm{O}_{\mathrm{w}}$ was determined using salinity and temperature data collected with depth for each site (research cruises AMT20, AMT24, SN105). However, for PG0 sites, these data were not available. Therefore, seawater temperature and salinity profiles for these regions collected at a comparable time of year to the specimens analysed were extracted from the World Ocean Database (Boyer et al. 2013). For example, specimens were collected at station PG0-78 $\left(26.62^{\circ} \mathrm{N}, 34.72^{\circ} \mathrm{E}\right)$ on 7 October 1988 , and temperature and salinity data were collected in the same region $\left(26.60^{\circ} \mathrm{N}, 34.98^{\circ} \mathrm{E}\right)$ on 26 September 1975. The regional $\delta^{18} \mathrm{O}$-salinity relationships of LeGrande \& Schmidt (2006) were applied (for example, Eq. 1). $\delta^{18} \mathrm{O}_{\mathrm{w}}$ was then converted from Vienna Standard Mean Ocean Water (V-SMOW) to V-PDB by subtracting $0.27 \%$ (Hut 1987). The method of Grossman et al. (1986), which uses the equation of O'Neil et al. (1969) with a correction factor of $0.6 \%$ for aragonite-calcite fractionation, was applied to calculate the aragonite equilibrium $\left(\delta^{18} \mathrm{O}_{\text {eq }}\right)$ with depth for each station (Eq. 2). Because atlantids produce aragonite in equilibrium with the seawater, the $\delta^{18} \mathrm{O}$ of the specimen was directly compared to the $\delta^{18} \mathrm{O}_{\text {eq }}$ depth profiles to determine the depth of calcification.

$$
\begin{aligned}
\delta^{18} \mathrm{O}_{\mathrm{w}}= & (0.55 \times S)-18.98 \\
& (\text { for North Atlantic stations })-0.27 \\
\delta^{18} \mathrm{O}_{\text {eq }}= & {[(-4.38-\sqrt{ } \Delta) /(2 \times 0.1)]+\delta^{18} \mathrm{O}_{\mathrm{w}}-0.6 \% }
\end{aligned}
$$$$
\text { when: } \Delta=4.38^{2}-[(4 \times 0.1) \times(16.9-T)
$$

In these equations, $S$ is salinity and $T$ is temperature.

These calculations do not take into account that the specimens may have formed their shells throughout different seasons of the year. The $\delta^{18} \mathrm{O}_{\mathrm{w}}$ is a function of salinity and therefore can vary seasonally, particularly in regions of high river runoff. However, in the open ocean where atlantids live, and away from polar regions that are greatly influenced by ice formation and melting, salinity does not show considerable seasonal or annual variation (Zweng et al. 2013). Therefore, we assumed here that there have been negligible seasonal and/or inter-annual variations in the $\delta^{18} \mathrm{O}_{\mathrm{w}}$ at each station.

\section{RESULTS AND DISCUSSION}

\section{Depth distributions and vertical migrations}

Temporal and spatial sampling coverage of collection data was well spread, with 289 different time-depth points during the day (07:00-18:59 h) and 1092 different time-depth points during the night (19:00-06:59 h, Fig. 2). Spatial sampling included 409 time-depth points for the upper $99 \mathrm{~m}$, and $341,154,258,49,170$ and 120 time-depth points for depths of 100-199, 200-299, 300-399, 400-499 and 500-600 m, respectively (Fig. 2).

Most species have a good temporal coverage of sampling, with records available for the majority of hourly intervals. Only 4 species (Atlanta ariejansseni, A. californiensis, A. meteori and Protatlanta sculpta) have considerable gaps in sampling time, with $<50 \%$ coverage for the hourly intervals (Fig. 2). The lack of sampling for 3 of these species (A. ariejansseni, A. californiensis, $P$. sculpta) can be explained because they occupy small regions relative to other atlantid species and have only been recently described (or reinstated). It is uncertain why there is a lack of sampling records of A. meteori during hours of daylight. For the remaining species, 2 main patterns of depth distribution

Fig. 2. Collated depth and time data from publications and collections. White diamonds: the maximum collection depth of all plankton net samples for each species. Black shaded areas: the maximum depth at which specimens were caught. Grey shaded areas: shallowest depth (sometimes the only depth) at which specimens were caught for each hourly interval. Original data are presented in Table S1 in the Supplement at www.int-res.com/articles/suppl/m587p001_supp.xlsx 
Sampling coverage

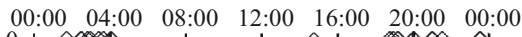

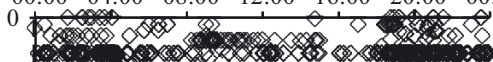

\& $-20 x$

$100 \div 000$

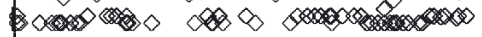

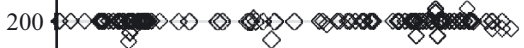

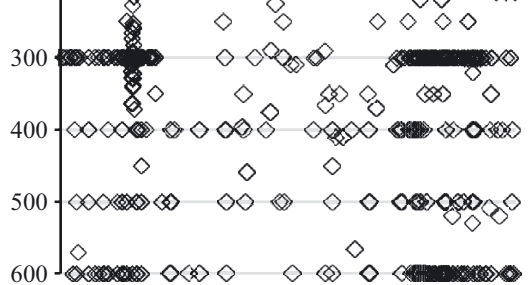

Atlanta californiensis

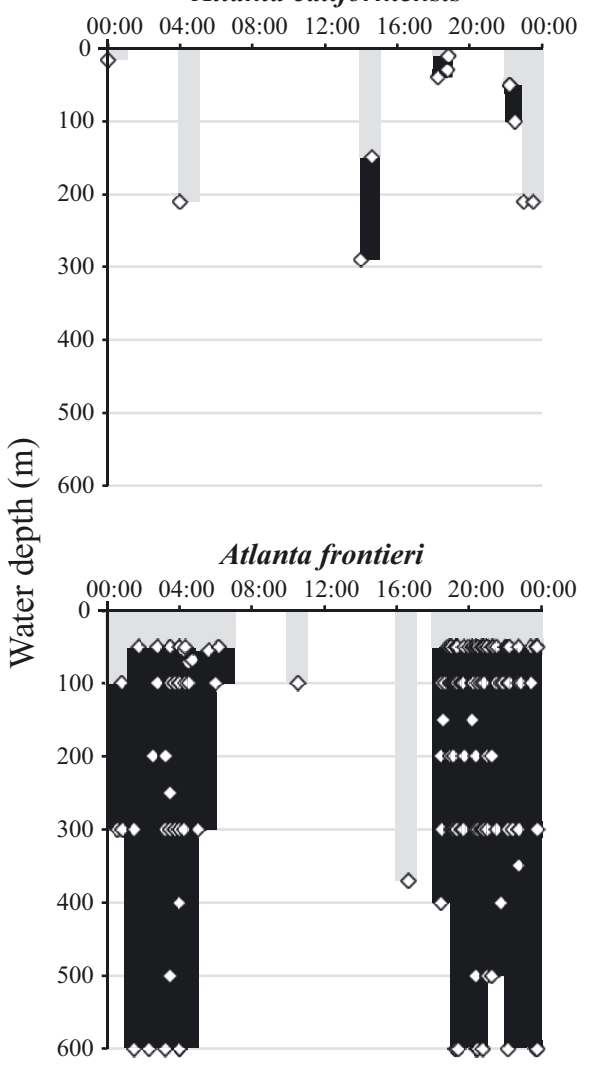

Atlanta helicinoidea

$\begin{array}{llllll}\text { 00:00 04:00 } & \text { 08:00 } & \text { 12:00 } & \text { 16:00 } & \text { 20:00 } & \text { 00:00 }\end{array}$

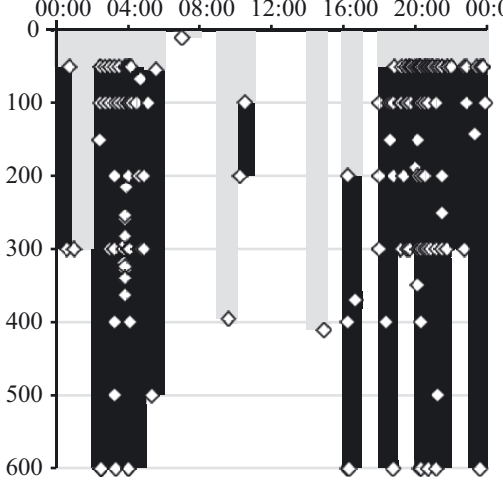

Time (h)

Atlanta ariejansseni

$\begin{array}{lllll}00: 00 & 04: 00 & 08: 00 & \text { 12:00 } & \text { 16:00 20:00 00:00 }\end{array}$

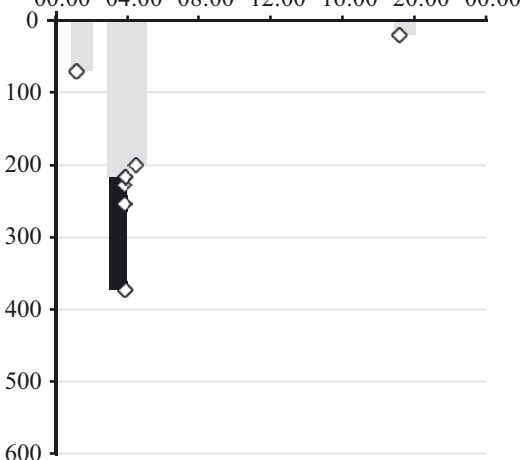

Atlanta echinogyra

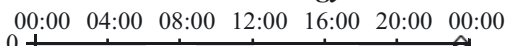

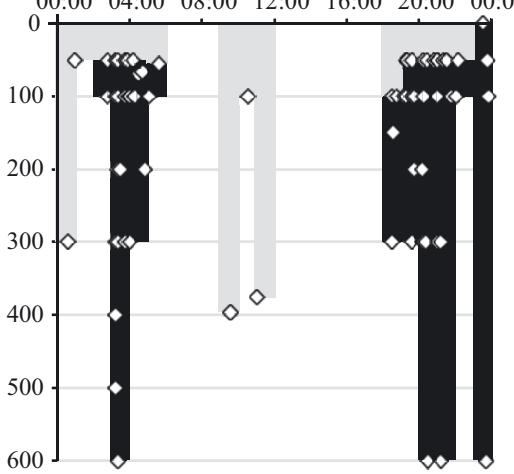

Atlanta gaudichaudi

$\begin{array}{lllll}00: 00 & 04: 00 & 08: 00 & 12: 00 & \text { 16:00 20:00 00:00 }\end{array}$

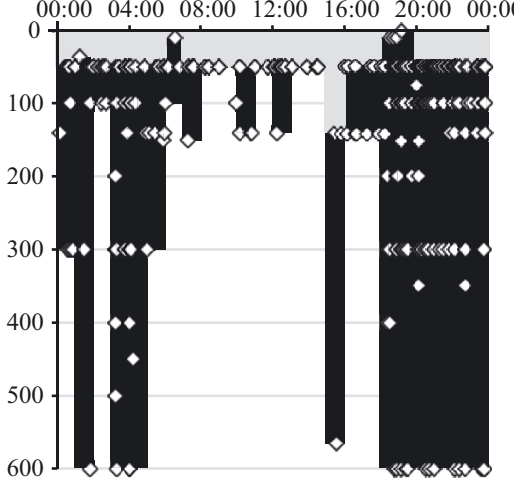

Atlanta inclinata

$\begin{array}{lllll}00: 00 & 04: 00 & 08: 00 & 12: 00 & \text { 16:00 20:00 00:00 }\end{array}$

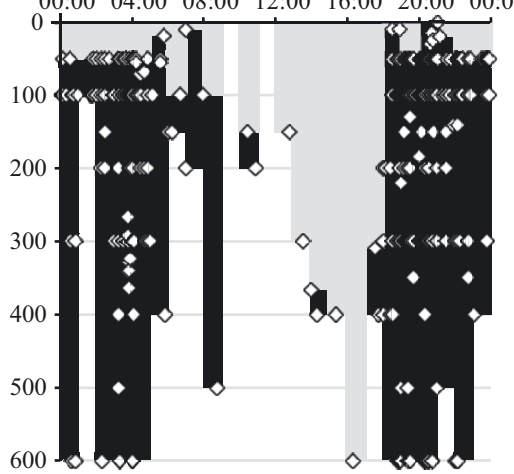

Atlanta brunnea

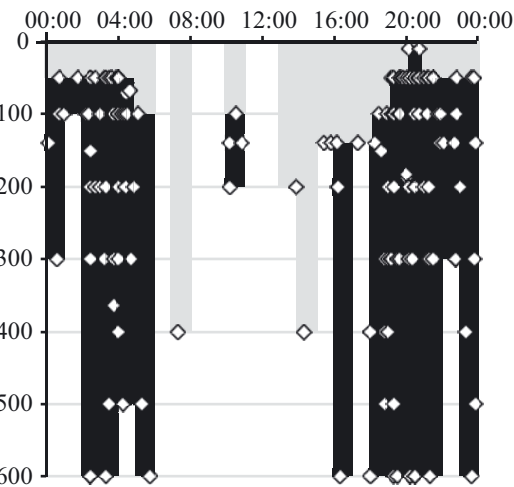

Atlanta fragilis

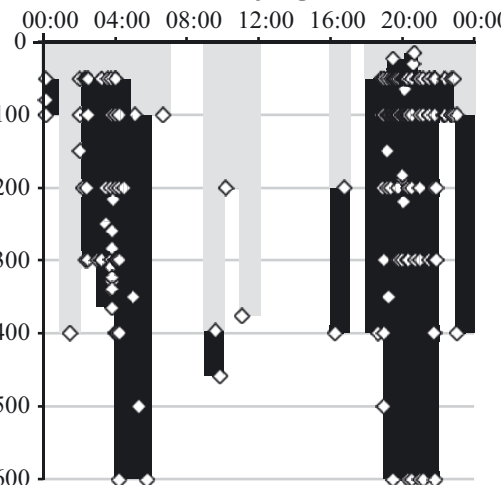

Atlanta gibbosa

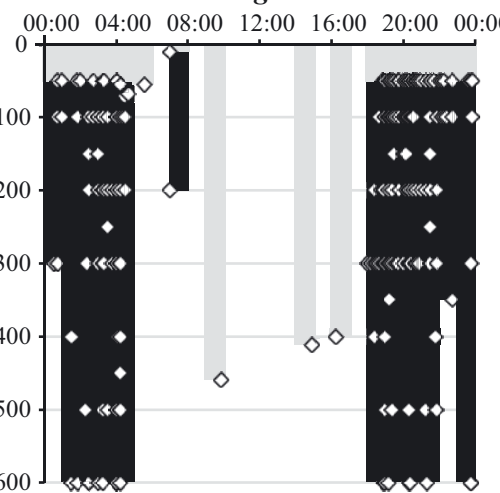

Atlanta inflata

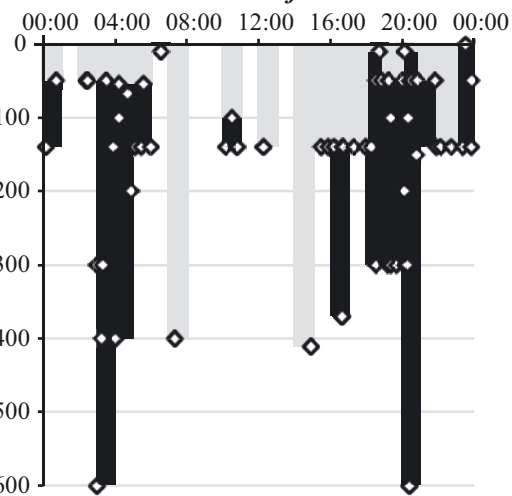

Fig. 2 continued on next page 
Time (h)

Atlanta lesueurii

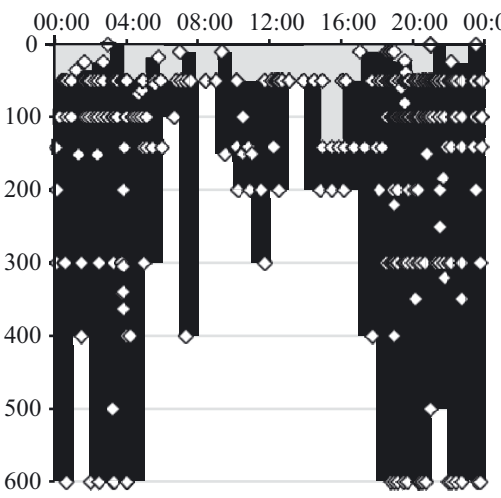

Atlanta peronii

$\begin{array}{lllllll}00: 00 & 04: 00 & 08: 00 & \text { 12:00 } & \text { 16:00 } & \text { 20:00 } & \text { 00:00 }\end{array}$

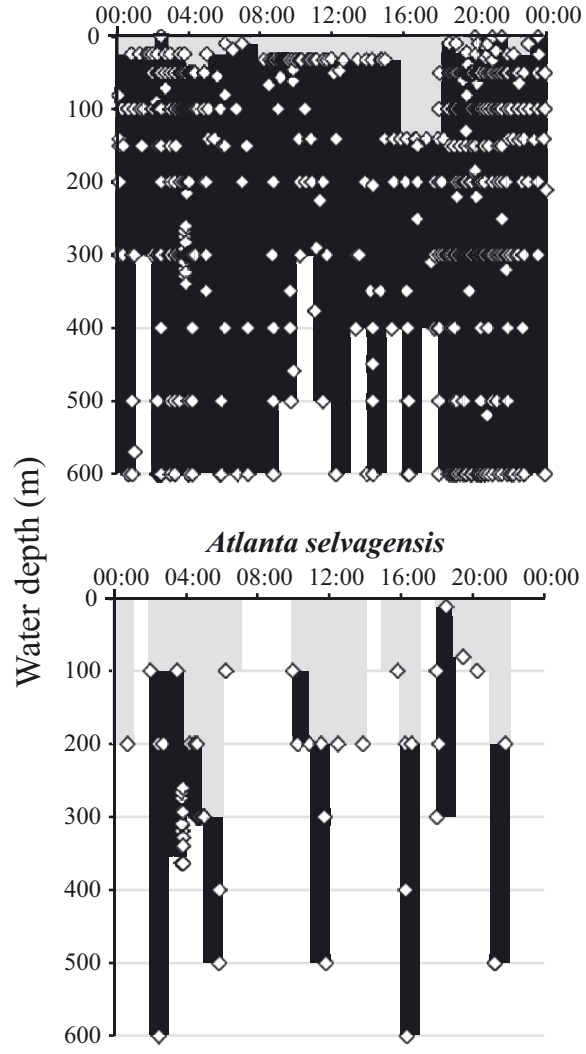

Oxygyrus inflatus

$\begin{array}{llllll}00: 00 & 04: 00 & 08: 00 & 12: 00 & \text { 16:00 20:00 00:00 }\end{array}$

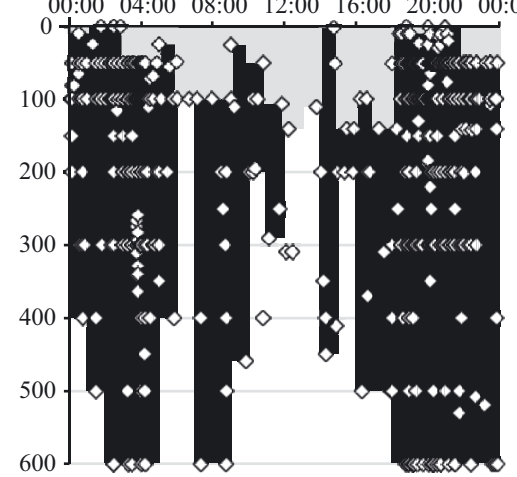

Atlanta meteori

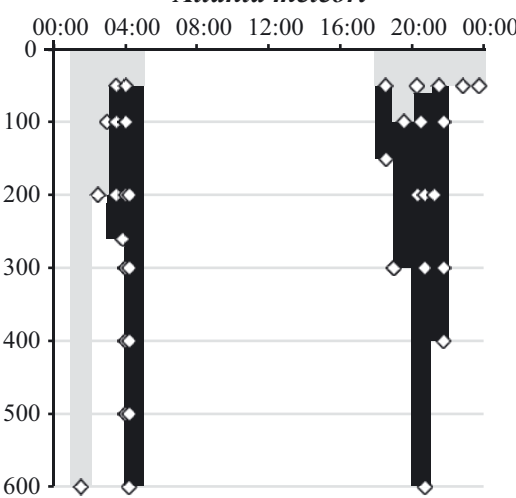

Atlanta plana

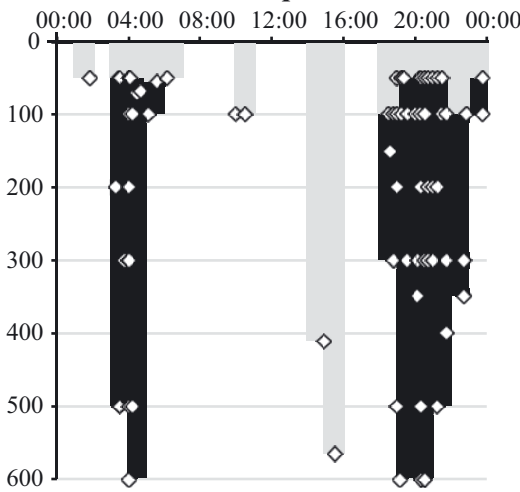

Atlanta tokiokai

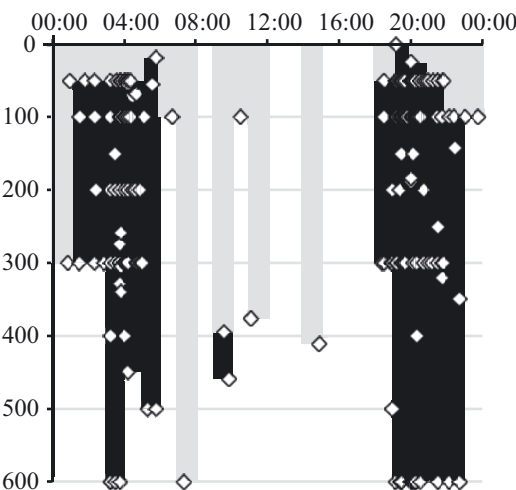

Protatlanta sculpta

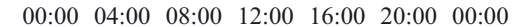

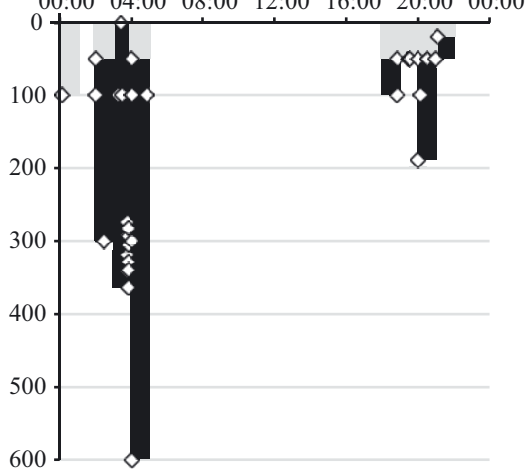

Atlanta oligogyra

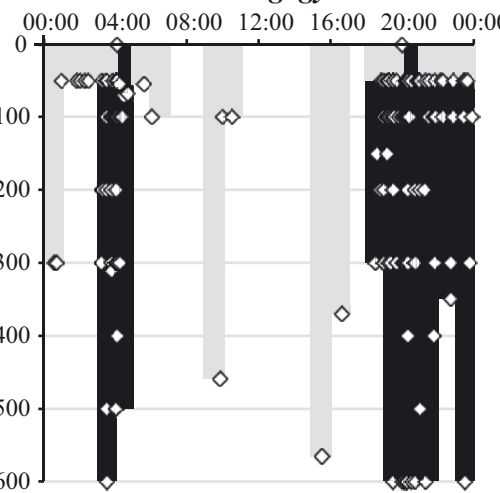

Atlanta rosea

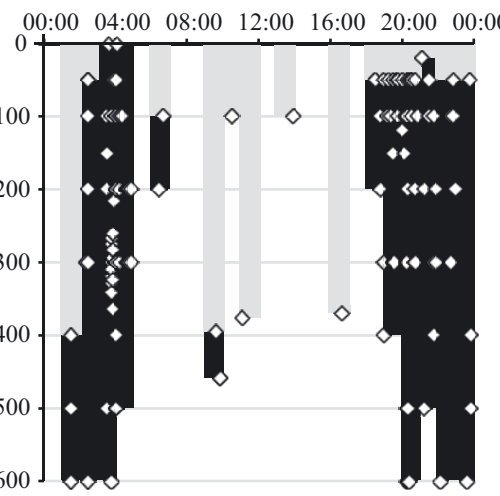

Atlanta turriculata

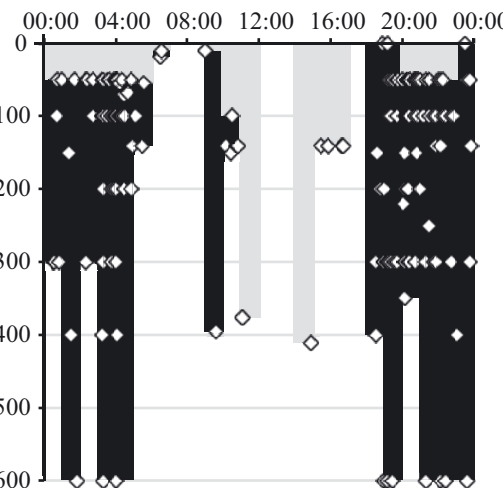

Protatlanta souleyeti

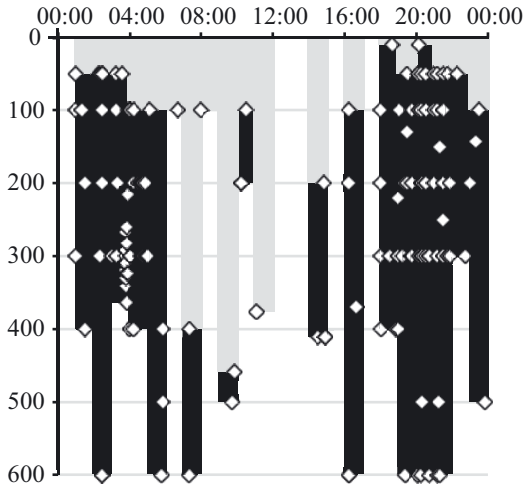

Fig. 2 (continued) 
with time were found. These are generally in agreement with the patterns of depth distribution found by Seapy (1990) offshore of Hawaii, i.e. species that remain in shallow waters at all times, and species that migrate to deeper waters during the daytime.

\section{Constant shallow water distribution}

Three species (A. peronii, A. gaudichaudi and A. lesueurii) did not undergo any diurnal migration, being present in shallow waters of $<50 \mathrm{~m}$ at most times (Fig. 2). The only deviation from this depth was between 15:00 and 18:00 $\mathrm{h}$ for all 3 species, when the maximum depth was $<140 \mathrm{~m}$. Although slightly deeper, A. selvagensis also showed a constant depth distribution, being present at $<200 \mathrm{~m}$ during the day and at night. Seapy (1990) and Lalli \& Gilmer (1989) also found that several smaller atlantid species were confined to shallow waters at all times. This supports the distributions of A. gaudichaudi, A. lesueurii and A. selvagensis. However, A. peronii, being one of the largest atlantid species, is generally considered to inhabit deeper waters $(>200 \mathrm{~m})$ and to be a diurnal migrator (Seapy 1990). Data presented here show that specimens were found in shallow water at all times (Fig. 2). Wall-Palmer et al. (in press) have shown that $A$. peronii is genetically diverse and may represent a collection of sub-species (or even species), each with a particular geographical distribution, and potentially each having a different depth distribution. Therefore, data presented here are probably a collation of all of these genetically different groups.

Two further species, A. inflata and Oxygyrus inflatus, were restricted to shallow water at all times, but also underwent a short-distance diurnal migration (Fig. 2). A. inflata showed a migration from $<50 \mathrm{~m}$ at night to $<140 \mathrm{~m}$ during the daytime; however, 2 periods of potentially deeper distribution occurred at 07:00-08:00 $\mathrm{h}$ and 14:00-15:00 $\mathrm{h}$ when the shallowest records were $<400 \mathrm{~m}$. O. inflatus varied in depth from $<50 \mathrm{~m}$ during the night to $<140 \mathrm{~m}$ during the day. Richter (1982) and Ossenbrügger (2010) also found $O$. inflatus to be restricted to the upper $100 \mathrm{~m}$, and most abundant in the upper $50 \mathrm{~m}$, and Seapy (1990) found $O$. inflatus to be restricted to the upper $90 \mathrm{~m}$ offshore of Oahu, Hawaii.

\section{Deep water diurnal migration}

Thirteen species displayed a consistent diurnal migration pattern (Fig. 2). These species had a shallow distribution, generally in the upper $50 \mathrm{~m}$, between $\sim$ 19:00 and 07:00 $\mathrm{h}$, and a deeper distribution, in at least the upper $100 \mathrm{~m}$, between 07:00 and 19:00 h. During parts of the daytime, each of these species was collected at depths of between 300 and $600 \mathrm{~m}$ but not in shallower water, despite the good temporal coverage of sampling at shallower depths. This suggests a diurnal migration that agrees with that proposed by several authors (Oberwimmer 1898, Seapy 1990, Michel \& Michel 1991, Seapy \& Richter 1993). However, a number of these species, including $A$. brunnea, A. helicinoidea and A. oligogyra were reported to be shallow non-migrators by Seapy (1990). None of the deep water migrating atlantid species exhibit the exact same migration path over time, reinforcing the view that migratory behaviour is species specific in atlantids (Richter 1973, Seapy 1990).

Despite the overall good spatial coverage of sampling points during the daytime, particularly in the upper $300 \mathrm{~m}$, many of the migrating species were only caught in very deep net samples during the daytime, well below the 250-300 m assumed maximum depth. For example, A. tokiokai and A. inclinata, 2 species with very similar shell morphology, were only caught in net samples of $>400 \mathrm{~m}$ during some time intervals during the daytime (Fig. 2). Some species were only caught at even greater depth, up to $1000 \mathrm{~m}$. Michel \& Michel (1991) also caught $A$. inclinata in openingclosing nets at a depth of 400-650 m during the daytime. These species are commonly caught in plankton samples (403 records presented here) and have a global distribution (Wall-Palmer et al. in press); therefore, if they were present in shallower waters during daylight hours, they would have likely been caught there. While it is improbable that atlantids descend into the aphotic zone (below $1000 \mathrm{~m}$ ), because their complex eyes determine that they rely on light, it is possible that they do migrate well below the euphotic zone $(200 \mathrm{~m})$ into the deeper waters of the dysphotic zone (200-1000 m). Previous studies have noted that larger atlantid species, some with larger eyes that may be adaptations to low light levels (e.g. A. fragilis, A. gibbosa), are often caught in deeper waters than smaller species. Seapy (1990) found that larger species offshore of Oahu migrated to deeper waters during the daytime. Similar trends of larger taxa with depth have been found in other mesopelagic zooplankton (Dai et al. 2017), and larger eyes relative to body size are thought to increase photon capture (and hence detection of predators and prey) in deeper dwelling mesopelagic fishes (de Busserolles et al. 2013).

Deep water migrations over $300 \mathrm{~m}$ suggest that some atlantids are exposed to large environmental 
gradients on a daily basis. The temperature difference from the ocean surface to $400 \mathrm{~m}$ is in the order of $5-20^{\circ} \mathrm{C}$ (from CTD data used in this study), far greater than the predicted anthropogenic change in ocean temperature. Even within the upper $200 \mathrm{~m}$, and particularly in the tropical regions, atlantids are experiencing variation in the aragonite saturation state of up to 2.5 units during diurnal migrations (Fig. 1). This means that deep migrating species may already be adapted to cope with large environmental changes. However, deep water migrating species may also be affected by the shallowing of the aragonite lysocline, which is predicted to be within a few hundred metres of the ocean surface globally by the year 2300 (Caldeira \& Wickett 2005, Orr et al. 2005, Raven et al. 2005). This will potentially alter atlantid migratory behaviour by reducing the depth to which they travel, and will undoubtedly have consequences for food availability, competition and predator interactions.

Nine of the deep water migrating species (A. echinogyra, A. frontieri, A. rosea, A. fragilis, A. helicinoidea, A. oligogyra, A. selvagensis, A. tokiokai and $P$. souleyeti), also display a brief deepening of their distribution at around midnight (Fig. 2). This phenomenon, described by Richter (1973) as the 'midnight sinking', has been observed by several authors (Oberwimmer 1898, Richter 1973, Seapy \& Richter
1993) and is thought to be caused by a lack of illumination which causes disorientation (Russell 1927). However, Richter (1973) found that the timing of this brief movement to deeper waters was species specific in atlantid larvae, being approximately $2 \mathrm{~h}$ after a species appeared in surface waters and not correlating with light conditions. This behaviour may therefore be more of a resting period during feeding, rather than a general disorientation caused by low light levels. Six species (A. brunnea, A. echinogyra, A. oligogyra, A. rosea, A. tokiokai and P. souleyeti) displayed a shallowing in their distribution at around 10:00-11:00 h, which has not been observed in previous studies. This could potentially be a period of swimming in between resting to avoid sinking into much deeper waters where environmental conditions (e.g. temperature, pH) are more hostile.

\section{Depth of calcification}

$\delta^{18} \mathrm{O}$ of atlantid heteropods yielded values between -0.32 and $-2.54 \%$ (Table 2, Fig. 3). In agreement with the findings of Grossman et al. (1986), the $\delta^{18} \mathrm{O}$ of specimens is comparable to the $\delta^{18} \mathrm{O}_{\text {eq }}$ for each station. The calculated depth of calcification is remarkably consistent between species and between sta-

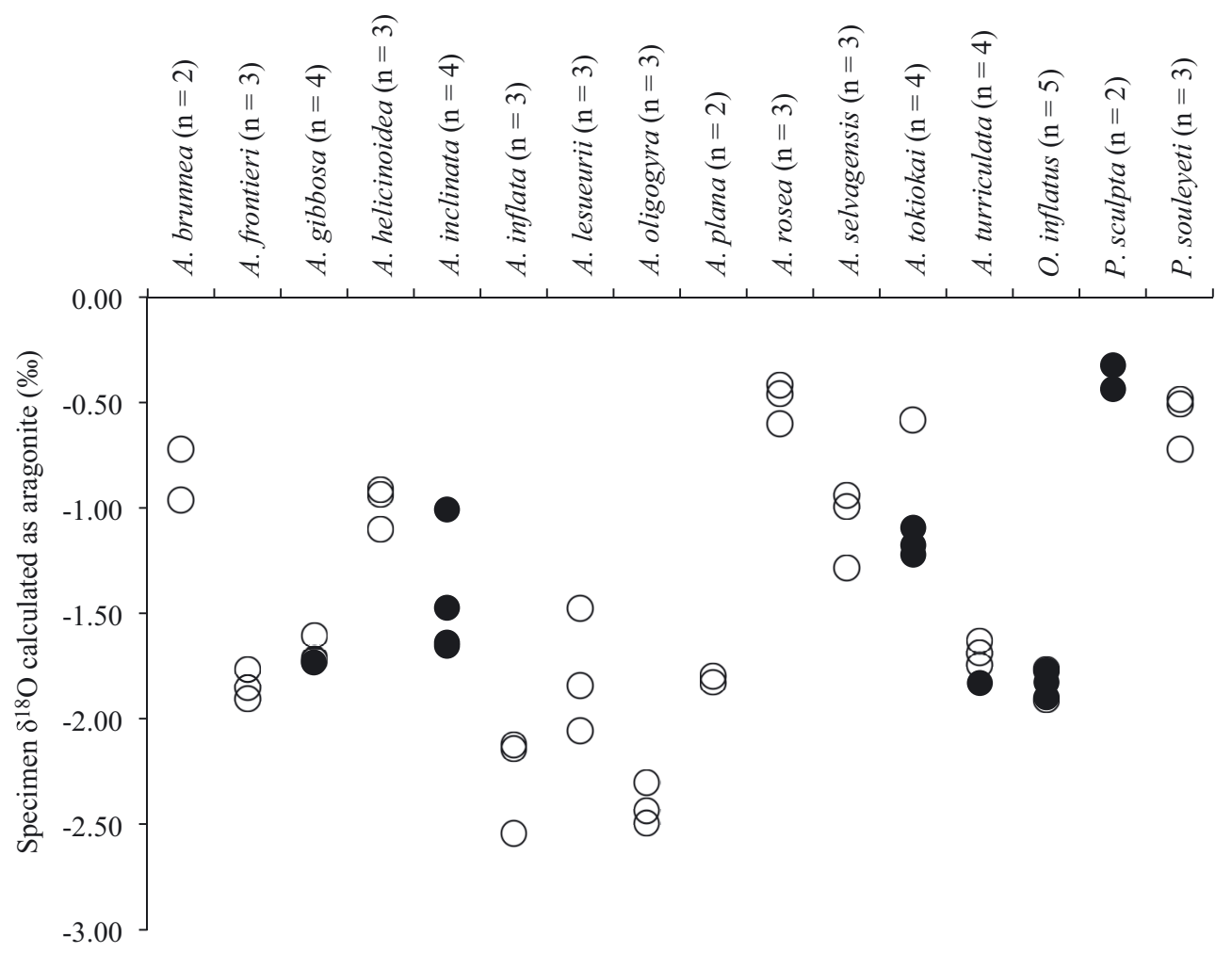

Fig. 3. Results of oxygen isotope analysis calculated as aragonite for all specimens. See Table 2 for values. These data include juvenile shells (filled circles) and adult shells (open circles). A. $=$ Atlanta $O .=$ Oxygyrus; $P .=$ Protatlanta 
tions (Fig. 4). All species, with the exception of $P$. souleyeti, were found to calcify in the upper 38-110 m. These depths are comparable to the analysis of Grossman et al. (1986), who found calcification depths of $75 \mathrm{~m}$ for $A$. inclinata and $A$. gaudichaudi. P. souleyeti produced a range of depths of 89.83-145.56 m.

The smaller species of Atlanta (adult shell 1.5-3 mm), including $A$. brunnea, A. helicinoidea, $A$. inflata, $A$. oligogyra and $A$. selvagensis, consistently calcify in shallower water $(38-79 \mathrm{~m})$ relative to larger species (adult shell 5-10 mm) such as A. frontieri, A. gibbosa, A. inclinata and $A$. rosea $(81-110 \mathrm{~m})$. A significant correlation was found between the average calcification depth and maximum adult shell size for species of the genus Atlanta (Fig. 5a, r = 0.66, p = $0.015, \mathrm{n}=13$ ). Calcification for all specimens, across all species and locations, was found to take place within the thermohalocline. For stations with chlo-

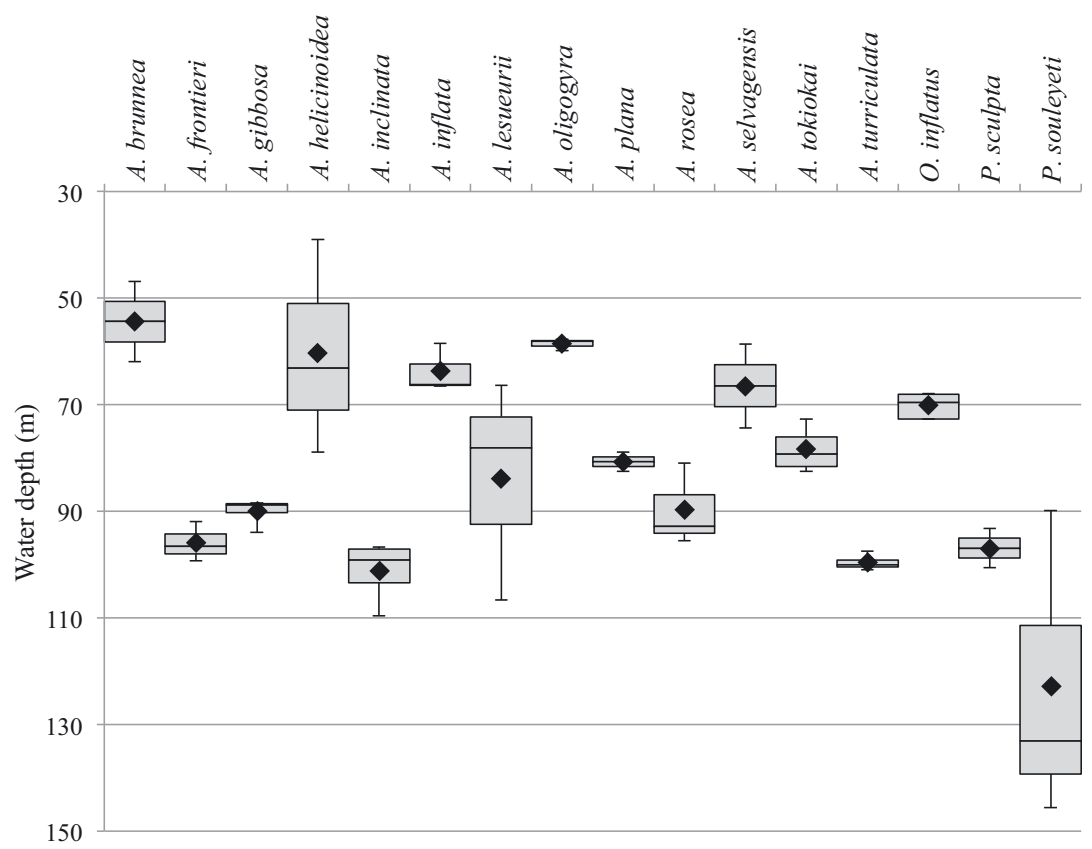

Fig. 4. Depth of calcification calculated using stable oxygen isotope analysis of atlantid shells, compared to the aragoniteseawater equilibrium (determined using seawater temperature and salinity). Original data are presented in Table 2. Black horizontal lines inside each box are the median depth, box limits are the first and third quartile, whiskers are the minimum and maximum depth, and black diamonds are the mean depth for each species. A. = Atlanta; O. = Oxagyrus; P. = Protatlanta
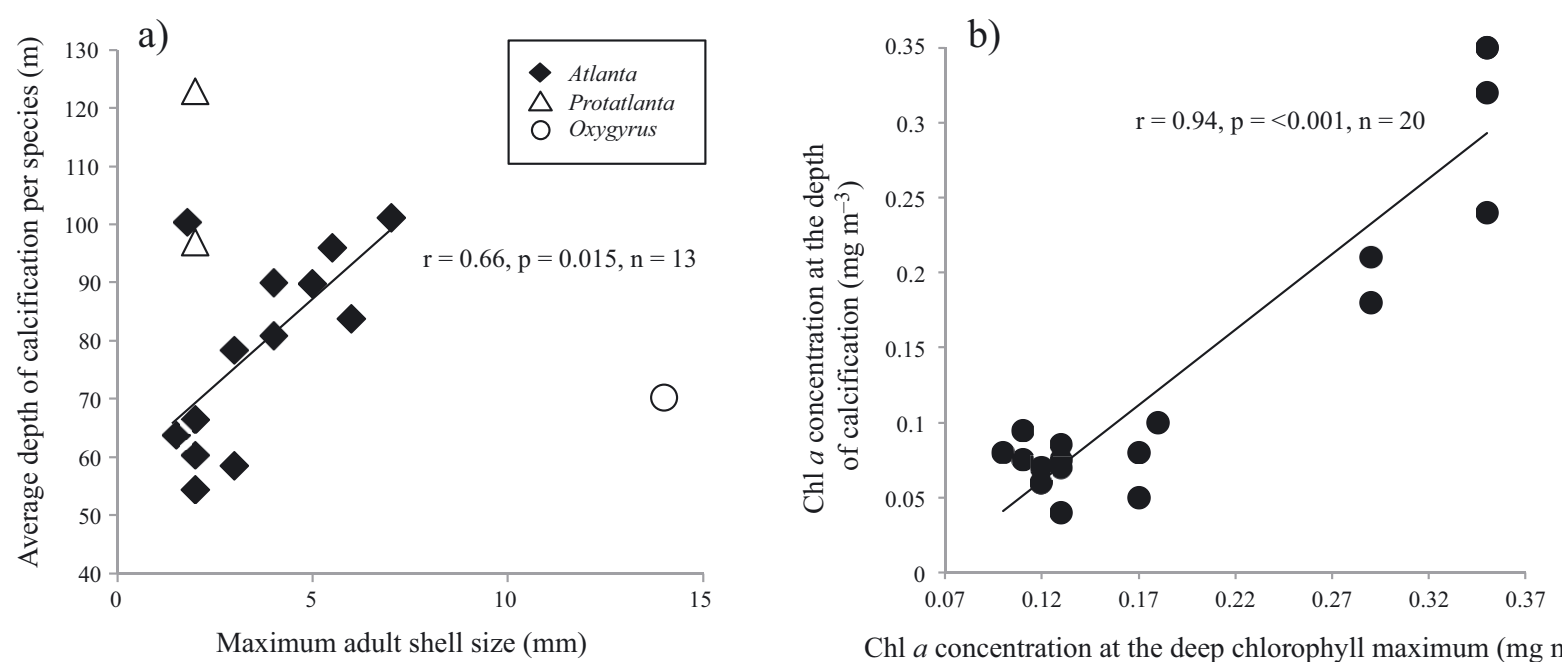

Fig. 5. (a) Average depth of calcification calculated for each species plotted against the maximum adult shell size for each species (Seapy 2011). Data for the genus Atlanta show a significant relationship. (b) Chlorophyll a concentration at the depth of calcification shows a significant relationship to the chlorophyll a concentration at the deep chlorophyll maximum (DCM) for specimens analysed from AMT20 and AMT24 stations 
rophyll-depth data (stations from research cruises AMT20 and AMT24), the concentration of chlorophyll $a$ at the depth of calcification was significantly correlated to the concentration of chlorophyll $a$ at the deep chlorophyll maximum (DCM, Figs. 5b \& 6). This could explain the deep calcification depth of $P$. souleyeti at stations AMT20-12 and AMT20-23 (133.06 and $145.56 \mathrm{~m}$, respectively), where the thermohalo-

Chlorophyll $a$ concentration $\left(\mathrm{mg} \mathrm{m}^{-3}\right)$
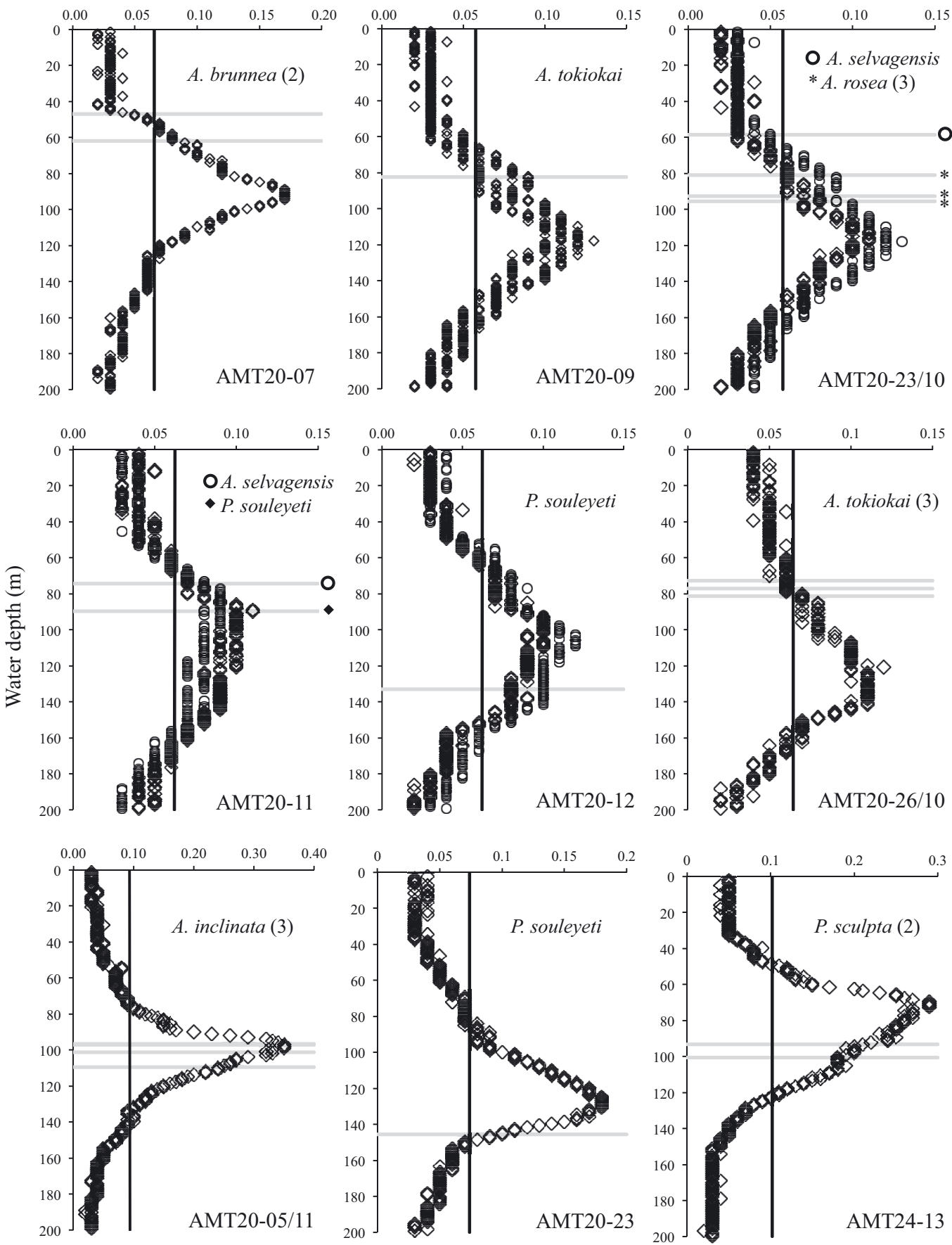

Fig. 6. Chlorophyll a concentrations over a water depth of $200 \mathrm{~m}$ for stations of Atlantic cruises AMT20 and AMT24 (data extracted from the British Oceanographic Data Centre). Black vertical lines: mean chlorophyll a concentration (over upper $200 \mathrm{~m}$ ). Horizontal grey lines: depth of calcification of atlantids calculated from the $\delta^{18} \mathrm{O}$ of shells, seawater temperature and salinity data. Where 2 species were collected from 1 station, the grey lines are labelled with symbols. Calcification takes place in regions with above mean chlorophyll a concentration, close to the deep chlorophyll maximum for most of the specimens analysed 
cline and DCM are deep relative to other stations. Preference to calcify within this nutrient-rich layer is likely linked to food availability. This is consistent with the findings of Keul et al. (2017), who demonstrated that the pteropod Heliconoides inflatus, a potential prey species for atlantids, calcifies at a depth of $\sim 75 \mathrm{~m}$ in the Atlantic Ocean.

Several of these data are based on whole adult shells; therefore, the calcifying depth is averaged over the entire life of the atlantid and may represent an average of deeper and shallower depths, as well as an average of different seasons and geographical areas. Particularly in adult specimens, shells may have been produced long before they were collected (e.g. in a different season when waters were warmer). The life cycle of atlantids is not well understood and the typical life span of an adult atlantid is unknown. The size and complexity of the velum in juvenile atlantids is characteristic of long-lived veligers (ThiriotQuiévreux 1973, Lalli \& Gilmer 1989) and may give an indication that their overall life span is long relative to other zooplankton groups. As a comparison, euthecosome pteropods are thought to have a life cycle of around 1.5-2 yr (Lalli \& Gilmer 1989). In this study, juvenile specimens were used for $30 \%$ of the $\delta^{18} \mathrm{O}$ analyses. In general, the depth of calcification in the juvenile stage (67.86 to $109.59 \mathrm{~m}$ ) was comparable to the depth of calcification for the adult shells (38.95 to $106.68 \mathrm{~m}$, excluding P. souleyeti) across different species and different geographical regions. For A. turriculata and A. gibbosa, both juvenile and adult shells were analysed for the same station. The $\delta^{18} \mathrm{O}$ (and depth of calcification) for both life stages was comparable (Table 2, Fig. 3). Grossman et al. (1986) also found no difference in the $\delta^{18} \mathrm{O}$ composition between 3 sections representing different life stages of a single Atlanta specimen. Therefore, although based here on a limited number of specimens, our results suggest that atlantids may calcify at the same depth throughout their lives (and stay within a small geographical region). This similarity between the $\delta^{18} \mathrm{O}$ of juvenile and adult shells could also have been produced if atlantids do not calcify at or close to the aragonite-water isotopic equilibrium throughout their lives, having varying metabolic effects on the isotopic composition throughout ontogeny-for example, if atlantids preferentially incorporate a higher proportion of $\mathrm{O}^{16}$ that originates from their own respired $\mathrm{CO}_{2}$ during shell growth. This has been found for some foraminiferan species (Pearson 2012). However, Grossman et al. (1986) found that the depths at which specimens were captured in sediment traps were in agreement with the depths at which the $\delta^{18} \mathrm{O}$ values of specimens were recorded, suggesting that there are very few or no metabolic effects upon specimen uptake of $\delta^{18} \mathrm{O}$.

\section{CONCLUSIONS}

This study combines collated collection data with stable oxygen isotope analysis of in situ collected material. Depth distribution data reveal 2 clear patterns of vertical distribution. The first pattern is of small atlantid species that reside in the upper $140 \mathrm{~m}$ of the ocean at all times and generally do not migrate. The second broad pattern of atlantid distribution, which the majority of species exhibit, is of larger species that carry out long diurnal migrations. During the night, these atlantids inhabit shallow waters, whereas during the daytime, they move to deeper waters. Some species may also move to very deep waters of over $300 \mathrm{~m}$, and several migrating species exhibit a 'midnight sinking' and/or a 'midday shallowing'.

The $\delta^{18} \mathrm{O}$ of the aragonite shells provides a first approximation to the depth of calcification, which appears to consistently represent a depth close to the DCM and within the upper $150 \mathrm{~m}$, for juvenile and adult specimens. This region is projected to undergo the earliest and greatest change in $\mathrm{pH}$ in response to increased anthropogenic $\mathrm{CO}_{2}$ and strongly indicates that atlantid heteropods will be adversely affected by ocean acidification of surface waters in the near future.

Acknowledgements. We are grateful to Alice K. Burridge (Naturalis Biodiversity Center, Leiden), Erica Goetze (University of Hawai'i at Mānoa) and Plymouth Marine Laboratory for providing samples and data from AMT24 and AMT20, respectively. We also thank Atushi Tsuda (University of Tokyo), Erica Goetze, Nina Bednaršek (Southern California Coastal Waters Research Project) and Lisette Mekkes (Naturalis Biodiversity Center) for providing data for cruises KH1110, VANC10MV and NOAA cruise WCOA16. We thank Tom Schiøtte, Martin Vinther Sørensen, Jon Ablett, Andreia Salvador and Joan Pickering for providing collections data and facilitating access to collections held at the Natural History Museum of Denmark and the Natural History Museum, London. We are grateful to Nina Keul and 2 anonymous reviewers for comments and suggestions on our manuscript. We acknowledge the scientists, captains and crews who took part in cruises AMT20, AMT24, PG0, SN105 (IIOE-2) and SO255, and the Atlantic Meridional Transect (AMT) programme. The SN105 expedition is part of IIOE-2 and was funded by the Indian National Centre for Ocean Information Services (INCOIS), Ministry of Earth Sciences, Govt. of India. The RV 'Sonne' cruise SO255 was funded by the German Federal Ministry of Education and Research (BMBF; grant 03G0255A). The 'Snellius II' Expedition (1984-1985) was 
organised by both the Netherlands Council of Oceanic Research and the Indonesian Institute of Sciences and received NWO funding (NWO-76-84 Urg.). This study is a contribution to the international IMBeR project, and plankton collection on the AMT20 and AMT24 cruises was partially supported by the UK Natural Environment Research Council (NERC) National Capability funding to Plymouth Marine Laboratory and the National Oceanography Centre, Southampton. This is contribution number 314 of the AMT programme. This work was supported by the Leverhulme Trust (RPG-2013-363, 2014-2017, to D.W.P., C.W.S), a NERC Isotope Geosciences Laboratory Grant (to D.W.P., C.W.S., M.J.L.) and a grant from the European Commission's (FP6) Integrated Infrastructure Initiative programme SYNTHESYS (DK-TAF) (to D.W.P.). This project has received funding from the European Union's Horizon 2020 research and innovation programme under the Marie Skłodowska-Curie grant agreement No. 746186 (POSEIDoN, D.W.P.). Access to aragonite saturation data was provided via an upload to the NOAA National Center for Environmental Information data portal.

\section{LITERATURE CITED}

Ayón P, Criales-Hernandez MI, Schwamborn R, Hirche HJ (2008) Zooplankton research off Peru: a review. Prog Oceanogr 79:238-255

Bednaršek N, Ohman MD (2015) Changes in pteropod distributions and shell dissolution across a frontal system in the California Current System. Mar Ecol Prog Ser 523: 93-103

Boyer TP, Antonov JI, Baranova OK, Coleman C and others (2013) World Ocean Database 2013. NOAA Atlas NESDIS 72 (Levitus S (ed), Mishonov A (tech ed). Silver Spring, MD

Burridge AK, Goetze E, Wall-Palmer D, Le Double SL, Huisman J, Peijnenburg KTCA (2017) Diversity and abundance of pteropods and heteropods along a latitudinal gradient across the Atlantic Ocean. Prog Oceanogr 158: 213-223

Caldeira K, Wickett ME (2005) Ocean model predictions of chemistry changes from carbon dioxide emissions to the atmosphere and ocean. J Geophys Res 110:C09S04

Craig H (1957) Isotopic standards for carbon and oxygen and correction factors for mass spectrometric analysis. Geochim Cosmochim Acta 12:133-149

Dai L, Li C, Tao Z, Yang G, Wang X, Zhu M (2017) Zooplankton abundance, biovolume and size spectra down to $3000 \mathrm{~m}$ depth in the western tropical North Pacific during autumn 2014. Deep Sea Res I 121:1-13

de Busserolles F, Fitzpatrick JL, Paxton JR, Marshall NJ, Collin SP (2013) Eye-size variability in deep-sea lanternfishes (Myctophidae): an ecological and phylogenetic study. PLOS ONE 8:e58519,

De Vera A, Seapy RR (2006) Atlanta selvagensis, a new species of heteropod mollusc from the Northeastern Atlantic Ocean (Gastropoda: Carinarioidea). Vieraea 34: 45-54

De Vera A, Seapy RR, Hernández F (2006) Heteropod molluscs from waters around the Selvagens Islands (Gastropoda: Carinarioidea). Vieraea 34:33-43

Furnestin ML (1961) Pteropodes et heteropodes du plancton morocain. Rev Trav Inst Peches Marit 25:293-326

Grossman EL, Ku TL (1986) Oxygen and carbon isotope fractionation in biogenic aragonite: temperature effects. Chem Geol 59:59-74

Grossman EL, Betzer PR, Dudley WC, Dunbar RB (1986) Stable isotopic variation in pteropods and atlantids from north Pacific sediment traps. Mar Micropaleontol 10:9-22

Hernández MP, Ferrandis E, Lozano Soldevilla F (1993) Pteropoda Thecosomata y Heteropoda (Mollusca, Gastropoda) en aguas del archipie'lago canario. Bol Inst Esp Oceanogr 9:263-283

Howard WR, Roberts D, Moy AD, Lindsay MCM, Hopcroft RR, Trull TW, Bray SG (2011) Distribution, abundance and seasonal flux of pteropods in the Sub-Antarctic Zone. Deep Sea Res II 58:2293-2300

Hut G (1987) Consultants group meeting on stable isotope reference samples for geochemical and hydrological investigations. IAEA, Vienna 16-18 September 1985. Report to the Director General, International Atomic Energy Agency. http://www.iaea.org/inis/collection/NCL CollectionStore/_Public/18/075/18075746.pdf

Jiang LQ, Feely RA (2015) Aragonite saturation state gridded to $1 \times 1$ degree latitude and longitude at depth levels of $0,50,100,200,500,1000,2000,3000$, and 4000 meters in the global ocean (NCEI Accession 0139360). Version 1.1. National Centers for Environmental Information, NESDIS, NOAA, US Department of Commerce, Washington, DC

Jivaluk J (1998) Distribution, abundance and composition of zooplankton in the South China Sea, Area II: Sabah, Sarawak and Brunei Darussalam waters. In: Proceedings of the Second Technical Seminar on Marine Fishery Resources Survey in the South China Sea, Kuala Lumpur, p 288-309

Juranek LW, Russell AD, Spero HJ (2003) Seasonal oxygen and carbon isotope variability in euthecosomatous pteropods from the Sargasso Sea. Deep Sea Res I 50: 231-245

Keul N, Peijnenburg KTCA, Anderson N, Kitidis V, Goetze E, Schneider RR (2017) Pteropods are excellent recorders of surface temperature and carbonate ion concentration. Sci Rep 7:12645

Lalli CM, Gilmer RW (1989) Pelagic snails: the biology of holoplanktonic mollusks. Stanford University Press, Stanford, CA

KLeGrande AN, Schmidt GA (2006) Global gridded data set of the oxygen isotopic composition in seawater. Geophys Res Lett 33:L12604

Lemus-Santana E, Sanvicente-Añorve L, Hermoso-Salazar M, Flores-Coto C (2014) The holoplanktonic Mollusca from the southern Gulf of Mexico. Part 1: heteropods. Cah Biol Mar 55:229-239

Maas AE, Wishner KF, Siebel BA (2012) The metabolic response of pteropods to acidification reflects natural $\mathrm{CO}_{2}-$ exposure in oxygen minimum zones. Biogeosciences 9: 747-757

McGowan JA, Fraundorf VJ (1966) The relationship between size of net used and estimates of zooplankton diversity. Limnol Oceanogr 11:456-469

Michel HB, Michel JF (1991) Heteropod and thecosome (Mollusca: Gastropoda) macroplankton in the Florida Straits. Bull Mar Sci 49:562-574

Mucci A (1983) The solubility of calcite and aragonite in seawater at various salinities, temperatures, and one atmosphere total pressure. Am J Sci 283:780-799

O'Neil JR, Clayton RN, Mayeda TK (1969) Oxygen isotope 
fractionation in divalent metal carbonates. J Chem Phys 51:5547-5558

Oberwimmer A (1898) Zoologische Ergebnisse 10; Mollusken 2. Heteropoden und Pteropoden, Sinusigera, gesammelt von SM Schiff 'Pola' 1890-1894. Denkschr Kaiserl Akad Wiss Wien Math Naturwiss Classe 65:573-594

Orr JC, Fabry VJ, Aumont O, Bopp L and others (2005) Anthropogenic ocean acidification over the twenty-first century and its impact on calcifying organisms. Nature 437:681-686

Ossenbrügger H (2010) Distribution patterns of pelagic gastropods at the Cape Verde Islands. MSc thesis, Helmholtz Centre for Ocean Research, Kiel

Paulinose VT, Gopalakrishnan TC, Nair KKC, Aravindakshan PN (1992) Stratification of zooplankton in the northwestern Indian Ocean. In: Desai BN (ed) Oceanography of the Indian Ocean. Oxford and IBH Publishing Company, New Delhi, p 113-120

Pearson PN (2012) Oxygen isotopes in foraminifera: overview and historical review. Paleontol Soc Pap 8:1-38

Quesquén Liza RC (2005) Moluscos holoplanctonicos heteropoda y pteropoda colectados en Noviembre y Diciembre de 1996 en el mar peruano. PhD thesis, Universidad Ricardo Palma, Lima

Raven J, Caldeira K, Elderfield H, Hoegh-Guldberg $\mathrm{O}$ and others (2005) Ocean acidification due to increasing atmospheric carbon dioxide. Policy document 12/05. The Royal Society, London

Richter G (1973) Field and laboratory observations on the diurnal vertical migration of marine gastropod larvae. Neth J Sea Res 7:126-134

Richter G (1982) Mageninhaltsuntersuchungen an Oxygyrus keraudreni (Lesueur) (Atlantidae, Heteropoda). Beispiel einer Nahrungskette im tropischen Pelagial. Senckenb Marit 14:47-77

Russell FS (1927) The vertical distribution of plankton in the sea. Biol Rev Camb Philos Soc 2:213-262

Seapy RR (1990) Patterns of vertical distribution in epipelagic heteropod molluscs off Hawaii. Mar Ecol Prog Ser 60:235-246

Seapy RR (2011) Atlantidae, Tree of Life web project. www.

Editorial responsibility: Antonio Bode,

A Coruña, Spain
tolweb.org/Atlantidae (accessed 15 December 2017)

Seapy RR, Richter G (1993) Atlanta californiensis, a new species of atlantid heteropod (Mollusca: Gastropoda) from the California Current. Veliger 36:389-398

Serrano O, Serrano L, Mateo MA (2008) Effects of sample pre-treatment on the $\delta^{13} \mathrm{C}$ and $\delta^{18} \mathrm{O}$ values of living benthic foraminifera. Chem Geol 257:218-220

Taki I, Okutani T (1962) Reports on the biology of the 'Umitaka-Maru' expedition, Part 2. J Fac Fish Anim Husbandry Hiroshima Univ 4:81-97

* Tesch JJ (1910) Pteropoda and Heteropoda. The Percy Sladen Trust Expedition to the Indian Ocean, 1905. Trans Linn Soc Lond 14:165-192

Thiriot-Quiévreux C (1973) Heteropoda. Oceanogr Mar Biol Annu Rev 11:237-261

Tokioka T (1955) On some plankton animals collected by the Syunkotu-Maru in May-June 1954. Publ Seto Mar Biol Lab 4:227-236

Van der Spoel S, Troost DG (1972) Atlanta tokiokai, a new heteropod (Gastropoda). Basteria 36:1-30

Wall-Palmer D, Burridge AK, Peijnenburg KTCA (2016a) Atlanta ariejansseni, a new species of shelled heteropod from the Southern Subtropical Convergence Zone (Gastropoda, Pterotracheoidea). ZooKeys 604:13-30

Wall-Palmer D, Burridge AK, Peijnenburg KTCA, Janssen AW, Kirby R, Hart MB, Smart CW (2016b) Evidence for the validity of Protatlanta sculpta (Gastropoda: Pterotracheoidea). Contrib Zool 85:423-435

Wall-Palmer D, Smart CW, Kirby R, Hart MB, Peijnenburg KTCA, Janssen AW (2016c) A review of the ecology, palaeontology and distribution of atlantid heteropods (Caenogastropoda: Pterotracheoidea: Atlantidae). J Molluscan Stud 82:221-234

Wall-Palmer D, Burridge AK, Goetze E, Stokvis FR and others (in press) Global biogeography and genetic diversity of atlantid heteropods. Prog Oceanogr, doi:10.1016/ j.pocean.2017.11.004

Zweng MM, Reagan JR, Antonov JI, Locarnini RA and others (2013) World Ocean Atlas, Vol 2: Salinity. NOAA Atlas NESDIS 74 (Levitus S (ed), Mishonov A (tech ed)). Silver Spring, MD

Submitted: September 18, 2017; Accepted: December 20, 2017 Proofs received from author(s): January 19, 2018 